
will undergo copyediting and typesetting before its final form for publication. We are providing this version as a service to our readers. The published version will differ from this one as a result of linguistic and technical

\title{
Comparison of the Anti-Obesity Effect of Enriched Capsanthin and Capsaicin from Capsicum annuum L. Fruit in Obesity-Induced C57BL/6J Mouse Model
}

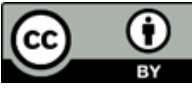

\section{SUMMARY}

Research background. Obesity increases mortality and morbidity due to its impact on type-2 diabetes, cardiovascular and gastrointestinal diseases, arthritis, and certain cancers. The epidemic of over mass and obesity requires constant endeavor research for improved therapies without undesirable side effects. Therefore, exploring the anti-obesity phytochemicals from food sources is essential. Most pharmacological studies of the anti-obesity potential of Capsicum annuum have been directed with capsaicin and very few with capsanthin. However, these studies utilized uncoated capsaicin and capsanthin. This study was directed to compare the anti-obesity effects of enteric-coated capsaicin and capsanthin in a high-fat diet-induced animal model.

Experimental approach. In the current study, we investigated the anti-obesity properties of capsanthin-enriched extract pellets and capsaicin pellets derived from red chili fruit (Capsicum annuum) on high-fat diet (HFD)-induced C57BL/6J obese mice. First, the animals were provided with HFD to induce their obesity. Then, oral supplementation of the test items was provided. The food intake, body mass, and obesity, as well as the clinical biomarkers, were assessed.

Results and conclusions. The mice fed with HFD were observed to gain body mass and white adipose tissue mass compared to the mice that consumed a normal diet. The oral administration of capsanthin-enriched extract pellets and capsaicin pellets significantly reduced the body mass gain.

\footnotetext{
*Corresponding author:

E-mail: astvel@gmail.com
} 
Please note that this is an unedited version of the manuscript that has been accepted for publication. This version will undergo copyediting and typesetting before its final form for publication. We are providing this version as a service to our readers. The published version will differ from this one as a result of linguistic and technical corrections and layout editing

Capsanthin-enriched extract pellets and capsaicin pellets have a statistically significant $(p<0.05)$ impact on obesity biomarkers by increasing adiponectin and decreasing leptin, free fatty acids, and insulin levels relative to HFD control. There was no change in the liver mass in all groups, but there was a significant decrease in white adipose tissues. Inguinal adipose tissue reduced by $37.0 \%$ and epididymal adipose tissue reduced by $43.64 \%$ after treatment with capsanthin-enriched extract pellets. These results suggest that capsanthin-enriched extract pellets and capsaicin pellets may be useful in combating metabolic diseases, including obesity, without adverse effects.

Novelty and scientific contribution. We increased the content of capsanthin greater than $50 \%$ in capsanthin-enriched extract crystals and enhanced the room temperature stability for more than one year by converting the crystals into capsanthin-enriched extract pellets. This study breaks new ground by examining the potential of capsanthin $>50 \%$ in the management of obesity for the first time.

Keywords: capsanthin-enriched extract pellets; capsaicin pellets; anti-obesity effect; high-fat diet

\section{INTRODUCTION}

Obesity alone is associated with an increased risk of morbidity and mortality (1), but with the COVID-19 pandemic, the condition has more severe consequences. Obesity is an important associated risk factor of mortality in people infected by COVID-19 (2) due to the virus' possible long-term, persistent, and irreversible undesirable effects on obesity (3). With the new pandemic, obesity might impact most cardiovascular disease (CVD) risk factors, including hypertension, diabetes mellitus, and abnormalities in blood glucose with undesirable effects on the cardiovascular system (4). Chronic inflammatory pulmonary disease and bronchial asthma are additional morbidities associated with obesity (5). The potential of having type-2 diabetes and almost all forms of CVD, especially heart failure, hypertension, coronary heart disease, atrial fibrillation, and peripheral arterial diseases (6), is increased due to the rise in central adiposity with visceral adipose tissue (7). Adipose tissues are responsible for energy storage and function and act as an endocrine organ known to secrete adipokines. The pro and anti-inflammatory activities of adipokines are linked to obesity related complications (8).

In recent years, the demand for dietary antioxidants, especially phytochemicals, has tremendously increased owing to their beneficial role in adiposity (9). To treat obesity, although various kinds of anti-obesity medications are available, these are associated with severe side effects (10). Therefore, exploring new phytochemicals with the potential to combat obesity without undesirable side effects is vital. The beneficial role of carotenoids in the management of obesity has been studied using 
Please note that this is an unedited version of the manuscript that has been accepted for publication. This version will undergo copyediting and typesetting before its final form for publication. We are providing this version as a service to our readers. The published version will differ from this one as a result of linguistic and technical corrections and layout editing

a natural carotenoid mixture and vitamins (11). The most studied carotenoids in lipid metabolism are $\beta$ carotene, cryptoxanthin, astaxanthin, and fucoxanthin; moreover, recently, capsanthin has been gaining more attention. In the prevention of excess adiposity and obesity, the role played by carotenoids and carotenoids derivatives is phenomenal (12). Carotenoids are highly unstable, especially in higher purity. Carotenoids degradation is followed by oxidation due to its characteristic conjugated polyene chain present its structure. Sometimes, the terminal groups also suffer degradation in certain environmental conditions. They also require storage under nitrogen and refrigerated conditions for their assured shelf life.

Therefore, the engineering of carotenoids to ensure stability is crucial (13). In order to maintain stability throughout their shelf life, encapsulation by using various polymers as coating agents has emerged as an excellent technique (14).

Red bell pepper (Capsicum annuum L.) has a long history of use as a vegetable, food color, and hot flavoring agent and in traditional medicines (15). The effect of red paprika capsanthin on obesity in both animal and human models has been reported, but no study has used highly enriched capsanthin. For the first time, we enriched capsanthin above $50 \%$ in capsanthin-enriched extract crystals and enhanced the room temperature stability of capsanthin-enriched extract pellets for more than one year. Moreover, we evaluated the anti-obesity effect of capsanthin-enriched extract pellets and capsaicin pellets derived from Capsicum annuum fruit in a high-fat diet induced (HFD) obesity model using male C57BL6/J mice. The anti-obesity effects of the oral administration of capsanthin-enriched extract pellets in three dosage groups, viz. low (20.5 mg/kg bm), mid (40.1 mg/kg bm), and high (82 mg/kg bm), compared to capsaicin pellets in one dose group (16 mg/kg bm) was compared between no dose and disease control groups.

\section{MATERIALS AND METHOD}

\section{Chemicals}

Capsaicin pellets $2 \%$ enteric-coated pellets were provided by Phytosol India Pvt Ltd, Bangalore, India. Sodium alginate (Vivapharm®, Alginate-PHC1, Evonik, Landerneau, France), microcrystalline cellulose powder (Pharmacel 101, DFE pharma, Nörten-Hardenberg, Germany), and ethyl cellulose (MP-20, Asha cellulose Pvt Ltd, Abrama, Valsad, Gujarat, India) were used. Besides, n-hexane, ethyl acetate, isopropyl alcohol, polyvinyl pyrrolidine K-30, sucrose, and dodecyl sulfate sodium salt were procured from Sigma-Aldrich, Bangalore, India. Orlistat purity $97 \%$ was purchased from TCl chemicals, Chennai, India. Normal fat diet and high-fat diet from Research Diet Inc, New Brunswick, USA were 
Please note that this is an unedited version of the manuscript that has been accepted for publication. This version will undergo copyediting and typesetting before its final form for publication. We are providing this version as a service to our readers. The published version will differ from this one as a result of linguistic and technical corrections and layout editing.

used in this study. The reference standard capsanthin was purchased from Sigma-Aldrich, St. Louis, USA.

\section{Capsanthin-enriched extract crystals}

Capsicum annuum fruits were procured from the local market in Bangalore, India, dried, deseeded, and flaked. The flaked fruits were extracted with n-hexane (16), then filtered, and concentrated to obtain the oleoresin. The oleoresin was further purified by supercritical extraction and subjected to alkaline saponification. The saponified extract was washed with water and purified with ethyl acetate to obtain capsanthin-enriched extract crystals (17).

\section{Capsanthin-enriched extract pellets}

In a glass bowl, capsanthin-enriched extract crystals, sucrose, and microcrystalline cellulose were mixed geometrically, and $5 \% \mathrm{~m} / \mathrm{V}$ polyvinyl pyrrolidine $\mathrm{K}-30$ solution in isopropyl alcohol was added to it to form a coherent mass. Wet pellets from the coherent mass were prepared by using an axial screw twin-screw extruder (Anish Pharma Equip Pvt. Ltd. Nashik, India). The wet pellets were transferred to a spheronizer (18) and dried. Sodium alginate and ethyl cellulose barrier coating were done by using a fluidized bed coater with bottom spray (Anish Pharma Equip Pvt. Ltd. Nashik, India). The pellets were removed and cured at room temperature.

Physical, chemical and microbial analyses of capsanthin-enriched extract pellets

The physical, chemical and microbial analyses were performed by using the methods described in United States Pharmacopeia (USP). The solubility of the capsanthin-enriched extract pellets in alcohol and water was analyzed by using the USP method 561 (19) by using $1 \mathrm{~g}$ of the pellets. The particle size was determined by dry sieve analysis as per the method described in USP 786 (20). In this method, $5 \mathrm{~g}$ of pellets passed through US sieve number 30 . The loss on drying was determined by drying $1 \mathrm{~g}$ of pellets at $105^{\circ} \mathrm{C}$ to constant weight as per the USP 731 (21) procedure.

The Joint Ethical Committee on Food Additives (JECFA) method was employed to quantify capsanthin as well as carotenoids (22). As per the method, capsanthin was extracted using acetone, saponified, and subjected to high-performance liquid chromatography (HPLC). The HPLC chromatograph equipped with LC solution software (model LC2030C, i serious plus, Shimadzu, Kyoto, Japan) was used. The C18 reversed-phase column with the dimensions $250 \times 4.6 \mathrm{~mm}, 5$ microns was used. The binary gradient mobile phase consists of acetone $(A)$ and water $(B)$. The gradient program 
Please note that this is an unedited version of the manuscript that has been accepted for publication. This version will undergo copyediting and typesetting before its final form for publication. We are providing this version as a service to our readers. The published version will differ from this one as a result of linguistic and technical corrections and layout editing

was set at 0 to $5 \mathrm{~min}, 75 \% \mathrm{~B}, 5$ to $10 \mathrm{~min}, 75-95 \% \mathrm{~B}, 10$ to $17 \mathrm{~min}, 95 \% \mathrm{~B}, 17$ to $22 \mathrm{~min}, 95 \%$ to 100 $\% \mathrm{~B}, 22$ to $75 \mathrm{~min}, 100$ to $75 \% \mathrm{~B}$. The total flow rate was maintained at $1.2 \mathrm{~mL} / \mathrm{min}$, and peaks were detected at $474 \mathrm{~nm}$. The standard and samples were prepared in acetone and sonicated for $10 \mathrm{~min}$. The retention time of capsanthin in the standard was used to identify the capsanthin in capsanthinenriched extract crystals and pellets. Carotenoids were estimated by spectrophotometry. The UV spectrophotometer equipped with Lab solution software (model 1900i, Shimadzu, Kyoto, Japan) was used. The suitable quantity of pellets was dissolved in acetone and measured the absorbance at $462 \mathrm{~nm}$ (22).

The elemental impurities like lead, arsenic, cadmium and mercury were estimated by using USP 233 (23) method. In this method, inductively coupled plasma-optical emission spectroscopy (ICP-OES) equipment Agilent model 5510 (Agilent corporation, Santa Clara, USA) was used. The ICP-OES instrument conditions were plasma; Argon $15 \mathrm{~L} / \mathrm{min}$, aux gas 0.9-1.0 L/min and analysis mode 500$700 \mathrm{kPa}$, nebulizer gas: 0.7-1.2 L/min maintained.

The microbiological parameters of the pellets were also assessed by USP procedures. The total plate and yeast and mold count was determined by using $1 \mathrm{~g}$ of pellets as per the USP 2021 method (24). Pathogens such as Escherichia coli, Salmonella species and Staphylococcus aureus were analyzed by using $10 \mathrm{~g}$ of pellets as per the method USP 2022 (25). The Pseudomonas aeruginosa analysis was per performed as per USP method 62 (26) by using $10 \mathrm{~g}$ of sample.

\section{Dissolution study}

The in vitro release profile of capsanthin-enriched extract and pellets was quantified by using dissolution apparatus (model No. DS 8000, Lab India, Bangalore, India) and following the dissolution method described in United states pharmacopeia USP 711 method (27). The dissolution experiment adopted the paddle method with $900 \mathrm{~mL}$ of $\mathrm{pH}=6.8$ phosphate buffer with $1 \%$ sodium lauryl sulfate as the media, the rotating speed of $100 \mathrm{rpm}$ and temperature $37^{\circ} \mathrm{C}$ was adopted to determine the dissolution of capsanthin. About $5.0 \mathrm{~mL}$ of each sample was withdrawn at regular time intervals and was replaced by the fresh media The quantification of capsanthin was done by using the HPLC method described above.

\section{Stability study}

The stability study was conducted as per the ICH guidelines Q1A (R2). Optimized formulations of capsanthin-enriched extract pellets were filled in a low-density polyethylene bag, packed in HDPE 
Please note that this is an unedited version of the manuscript that has been accepted for publication. This version will undergo copyediting and typesetting before its final form for publication. We are providing this version as a service to our readers. The published version will differ from this one as a result of linguistic and technical corrections and layout editing.

containers. The containers were kept in a stability chamber, and temperature $(25 \pm 2){ }^{\circ} \mathrm{C}$ and relative humidity $(60 \pm 5) \%$ were maintained. The samples were analysed initially and after the end of 3-, 6-, 9, and 12-months' period (28). The following parameters were examined: change in the appearance such as colour, identification of capsanthin by HPLC, content of capsanthin, and carotenoids.

Isolation of pure capsanthin by preparative HPLC

A spectroscopically pure capsanthin was isolated from partially purified capsanthin-enriched extract crystals. The separation was performed with a preparative HPLC instrument (model Agilent 1200, Agilent Corporation, Santa Clara, USA). The pure capsanthin fraction was characterized by LCMS, ${ }^{1} \mathrm{H}-\mathrm{NMR}$, and ${ }^{13} \mathrm{C}-\mathrm{NMR}$.

\section{LC-MS analysis}

The preparative HPLC fraction 3 (F3) was analyzed to determine the mass by in-house developed LC-MS with a binary gradient elution using Waters LCMS Mircomass ZQ, quadrupole mass analyzer coupled with tandem HPLC (model No. 2695, Xevo G2-Xs QTof, Water's Corporation, Milford, USA).

${ }^{1} \mathrm{H}$ and ${ }^{13} \mathrm{C}$ NMR characterization

${ }^{1} \mathrm{H}$ and ${ }^{13} \mathrm{C}$ nuclear magnetic resonance spectra (29) were recorded to determine the structure for the isolated capsanthin on Agilent-NMR (model No. VNMRS-400, Agilent Corporation, Santa Clara, USA). The samples were prepared in deuterated chloroform $\left(\mathrm{CDCl}_{3}\right)$. The various proton environments were monitored by performing an $\mathrm{H}-\mathrm{H}$ correlation analysis within the chemical structure.

\section{Animals and experimental protocol}

The Institutional Animal Ethics Committee (IAEC) approved this in vivo study protocol vide approval number VIP/IAEC/156/2019. This study was conducted by implementing the measures recommended by the CPCSEA (Committee for Control and Supervision of Experiments on Animals) (30). C57BL/6 mice, four-week-old males (Hylasco, Hyderabad, India), were acclimatized for one week to a specific temperature $(22 \pm 3)^{\circ} \mathrm{C}$, humidity (30 to $\left.70 \pm 5\right) \%$, and lighting 08:00-20:00 conditions after their procurement. Polycarbonate cases were used to house the animals, and free access to drinking water and food was provided. The C57BL/6 mice were randomly divided into seven groups of ten mice each after adaptation. Group G1 (normal diet, ND) was fed with a $10 \%$ kcal fat diet, and the remaining 
Please note that this is an unedited version of the manuscript that has been accepted for publication. This version will undergo copyediting and typesetting before its final form for publication. We are providing this version as a service to our readers. The published version will differ from this one as a result of linguistic and technical corrections and layout editing.

groups (G2 to G7) were fed with a $60 \% \mathrm{kcal}$ fat diet to induce obesity for seven weeks. The induction of obesity was confirmed by body mass increase. The test item capsanthin-enriched extract pellets were suspended in $0.5 \%$ carboxymethyl cellulose (CMC) and administrated orally for the groups G3low (20.5 mg/kg/ bm), G4-mid (41 mg/kg/ bm), and G-5 high dose (82 mg/kg/ bm) for five weeks. The group G6 and G7 received capsaicin pellets (16 mg/kg/ bm) and orlistat (16 mg/kg/ bm) in 0.5\% CMC respectively for five weeks. The normal diet control (G1) and HFD control (G2) mice groups were provided with only $0.5 \%$ CMC. The body mass and food intake were measured twice weekly during the obesity induction period and thrice weekly thereafter up to the end of the treatment.

\section{Clinical pathology}

At the end of the study period, blood samples were collected for hematology, clinical pathology investigations, and biomarker analysis. The hematology analyzer (model ABX Micros ESV 60, HORIBA Instruments Inc. Irvine, USA) was used to perform a hematological evaluation at the end of the study period. Clinical chemistry parameters were estimated at the end of the study period by using the $R X$ Daytona+ (Randox Laboratories, Kearneysville, USA) instrument. Commercially available enzymelinked immunosorbent assay kits (Kinesis Dx kits, Krishgen Biosystems, Mumbai, India) were used to quantify insulin, leptin, adiponectin and free fatty acid. At the end of the study, all animals were subjected to a detailed gross necropsy examination. External body surfaces, orifices, and cavities such as abdominal, thoracic, and cranial were included in the examination.

\section{Histopathology and necropsy}

When the body mass was measured, the liver, abdominal adipose tissues (inguinal and white epididymal), and kidney tissues were fixed in $10 \%$ natural buffered formalin, stained with hematoxylin and eosin (Everon Life Sciences, New Delhi, India), and subjected to histopathological evaluation. Adipose tissues were observed microscopically for any change in adipocytes. All the animals were required to complete both internal and external gross necropsy examinations.

\section{Statistical analysis}

ANOVA, a one-way analysis of variance, was used for multiple comparisons. The effects of treatment compared to control were analyzed by using the GraphPad software, v. 8.0, California, USA (31). The values were expressed as mean \pm S.D. (standard deviation), compared, and evaluated at a 5 $\%$ ( $p \leq 0.05)$ level. 
Please note that this is an unedited version of the manuscript that has been accepted for publication. This version will undergo copyediting and typesetting before its final form for publication. We are providing this version as a service to our readers. The published version will differ from this one as a result of linguistic and technical corrections and layout editing

\section{RESULTS AND DISCUSSION}

In our study, capsanthin was enriched to above $50 \%$, which enhanced the room temperature storage stability to greater than one year by converting it into capsanthin-enriched extract pellets. The appearance of capsanthin-enriched extract crystals, capsanthin-enriched extract pellets, and capsaicin pellets is shown in Fig. S1. The pellet and coating compositions that have been employed are shown in Table S1, and the specification for capsanthin-enriched extract pellets is shown in Table S2. The capsanthin-enriched extract pellets comply with all the parameters mentioned in Table S2.

\section{In vitro drug release}

The hydrophilic matrix formulation of capsanthin-enriched extract pellets was developed using microcrystalline cellulose, sucrose, and polyvinyl pyrrolidine $\mathrm{K} 30$ and further barrier coated with sodium alginate and ethyl cellulose. The polymers on the exterior surface hydrate and swell, forming a protective gel layer and slowly and steadily releasing capsanthin over time. The release of capsanthin is due to either diffusion or erosion or the combination of both processes from the gel layer (32). The cumulative capsanthin release profiles of capsanthin-enriched extract crystals and capsanthin-enriched extract pellets using the dissolution apparatus USP type-2 in the $\mathrm{pH}=6.8$ buffer is shown in Fig. S2. As can be seen, the two types of capsanthin-enriched extract crystals and capsanthin-enriched extract pellets exhibited different release patterns. The release profile of capsanthin-enriched extract crystals was observed to be rapid, while that of capsanthin-enriched extract pellets was observed to occur in a sustained release manner. The in vitro release profile of capsanthin-enriched extract crystals and capsanthin-enriched extract pellets is shown in Fig. S2.

\section{Stability of capsanthin-enriched extract pellets}

The physical and chemical stability of capsanthin-enriched extract pellets was evaluated as per the international conference on Harmonization guidelines. Test samples of capsanthin-enriched extract pellets were stored at $25^{\circ} \mathrm{C}$ and $60 \%$ relative humidity for one year. The capsanthin-enriched extract pellet samples were packed in low-density polyethylene bags and enclosed in a miniature high-density polyethylene container. Physical and chemical stability evaluations were initially performed and after 3 , 6, 9, and 12 -months' storage periods. Organoleptic analysis was carried out to evaluate the physical stability. The chemical stability of the capsanthin-enriched extract pellets was determined through the HPLC method. In addition, loss on drying and the total carotenoids were quantified. At the end of one year, capsanthin-enriched extract pellets were stable at $25^{\circ} \mathrm{C}$ and $60 \%$ relative humidity. At the end of 
Please note that this is an unedited version of the manuscript that has been accepted for publication. This version will undergo copyediting and typesetting before its final form for publication. We are providing this version as a service to our readers. The published version will differ from this one as a result of linguistic and technical corrections and layout editing.

the study period, no physical and chemical changes were observed. The stability data are presented in Table S3.

\section{Spectral characterization}

LC-tandem mass spectrometry with HPLC technique was employed to determine the chemical profile of capsanthin-enriched extract. Liquid chromatogram patterns of capsanthin-enriched extract showed major peaks at their retention times of 5.88, 6.24, 6.84, and 7.11 (Fig. S3). Furthermore, capsanthin-enriched extract indicated a molecular ion peak at a retention time of 7.11 min with the fragmentation of $\mathrm{m} / \mathrm{z}=585.30[\mathrm{M}+]+($ Fig. S4) and dehydrated productions $([\mathrm{MH}-\mathrm{H} 2 \mathrm{O}]+$ at $\mathrm{m} / \mathrm{z}=567$, which was correlated to capsanthin from literature studies (33). In order to unequivocally prove the capsanthin identification, after isolation from capsanthin-enriched extract, the structure was confirmed using ${ }^{1} \mathrm{H}$-NMR and ${ }^{13} \mathrm{C}-\mathrm{NMR}$ spectroscopy. The NMR data were consistent with those in the literature. The ${ }^{1} \mathrm{H}-\mathrm{NMR},{ }^{13} \mathrm{C}-\mathrm{NMR}$ data, and the structure of capsanthin are shown in Fig. S5a, S5b and S5c respectively.

The anti-obesity effect of capsanthin-enriched extract pellets and capsaicin pellets on HFD-induced C57BL/6J mice

Effect of capsanthin-enriched extract pellets on body mass

The effect of capsanthin from red bell pepper on adipogenesis and mass gain inhibition in HFDinduced animal models was reported by Kim et al. (34) by using a low concentration of $0.005 \%$ capsanthin and Jo et al. (35) used a mixture of trans-esterified carotenoids with a maximum concentration of $10 \mu \mathrm{mol}$ capsanthin. In this study, the anti-obesity activity of capsanthin-enriched extract pellets (>50 \% capsanthin) on HFD-induced C57BL/6 mice was evaluated and compared to HFD control, capsaicin pellets $2 \%$, and the reference drug orlistat.

HFD-induced obesity was confirmed after the mice were fed with HFD for seven weeks. The HFD group showed a statistically significant body gain $(p<0.05)$, and the mean body mass gain was $164.1 \%$ compared to the ND group. A statistically significant $(p<0.05)$ mass gain reduction was observed in the HFD + capsanthin-enriched extract pellets and HFD + capsaicin pellets administered groups compared to the HFD control. The mass gain reduction in HFD + capsanthin-enriched extract pellets low-dose, mid-dose, and high-dose was $34.70 \%, 51.14 \%$, and $98.51 \%$ respectively. These values can be compared to the HFD + capsaicin pellets (57.15\% lower) and HFD + orlistat group (48.86 $\%$ lower). Our study validated the mass gain inhibition action of capsanthin in an animal model as 
Please note that this is an unedited version of the manuscript that has been accepted for publication. This version will undergo copyediting and typesetting before its final form for publication. We are providing this version as a service to our readers. The published version will differ from this one as a result of linguistic and technical corrections and layout editing

previously reported by Kim et al. (34) and Jo et al. (35) but could not compare the efficacy of our capsanthin to previously reported studies due to a lack of sufficient information and a statistically nonsignificant mass gain reduction reported by Jo et al. (35). Wu et al. (36) reported the mass gain reduction as $27.5 \%$ in the HFD-induced obese model but the study involved low pure capsanthin extract. A significantly higher food intake was observed in the ND group compared to the HFD group, but there was not much variation between the control and treated groups as shown in Figs. 1a and 1b respectively. The summary of food intake is shown in Table S4.

Effect of capsanthin-enriched extract pellets on HFD-induced changes in adiponectin levels in mice

Adiponectin is one of the adipokines, predominantly secreted by adipose tissues in adult humans, with insulin-sensitizing action through adiponectin receptors (Adipo R1 and Adipo R2) and anti-inflammatory and anti-apoptotic properties (37). Adiponectin enhances insulin sensitivity and signaling by activating $A M P K, P P A R-\gamma$, and other unknown pathways (38). In skeletal muscles, adiponectin increases $C D 36$ and acyl-coenzyme-A oxidase expression leads to a reduction in the free fatty acids and triglyceride levels (39). Furthermore, adiponectin ensures an increase in glucose uptake and fatty acid combustion leads to a reduction in blood glucose (40). In all HFD+capsanthin-enriched extract pellets treated groups; the adiponectin level was increased significantly $(p<0.05)$. The adiponectin level for HFD+capsanthin-enriched extract pellets low, mid, and high dose treated groups were $(2.65 \pm 0.47),(2.86 \pm 0.34)$, and $(2.77 \pm 0.44) \mu \mathrm{g} / \mathrm{mL}$ respectively, and for the HFD group, the adiponectin level was $(1.98 \pm 0.26) \mu \mathrm{g} / \mathrm{mL}$. A similar trend was observed in HFD+capsaicin pellets (2.64 \pm 0.44$) \mu \mathrm{g} / \mathrm{mL}$ as well as the HFD+orlistat group $(2.74 \pm 0.74) \mu \mathrm{g} / \mathrm{mL}$. Overall, in the HFD+capsanthin-enriched extract pellets high dose treated group, a significant increase in the adiponectin level (28.02 \% higher) was observed compared to the HFD control group and this is remarkable when compared to Kim et al. (8.65 \% increase) (34) and Jo et al. (statistically non-significant increase level) (35). The data are presented in Fig. 2a.

\section{Effect of capsanthin-enriched extract pellets on HFD-induced changes in leptin levels in mice}

Leptin, another adipokine that regulates energy expenditure and food intake, is encoded by the obese gene and is closely related to the adipose tissue mass and body mass index (BMI) in humans (41). In rodents and humans, a linear correlation was noted between leptin levels and mass loss. Elevated leptin level in plasma, which is the leading cause of obesity in humans. The plasma leptin level may be an indicator of body fat increase (42). Capsanthin reduces serum leptin level in HFD-induced 
Please note that this is an unedited version of the manuscript that has been accepted for publication. This version will undergo copyediting and typesetting before its final form for publication. We are providing this version as a service to our readers. The published version will differ from this one as a result of linguistic and technical corrections and layout editing

animal model as reported by Kim et al. (34), which is confirmed by our study. The high-fat diet significantly increased $(p<0.05)$ the blood plasma leptin level compared to control group. The leptin levels for HFD and ND groups were $(301.99 \pm 25.60)$ and $(137.98 \pm 22.68) \mathrm{pg} / \mathrm{mL}$ respectively. A statistically significant $(p<0.05)$ decrease of leptin was observed in all HFD+capsanthin-enriched extract pellets treated groups compared to HFD control. The leptin level for capsanthin-enriched extract pellets

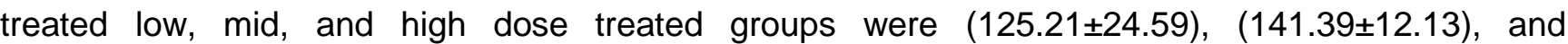
(153.92 \pm 33.68$) \mathrm{pg} / \mathrm{mL}$ respectively. In the same manner, the HFD+capsaicin pellets treated group and HFD+orlistat treated group showed a statistically significant decrease in leptin levels compared to the HFD control group. The leptin level for the HFD+capsaicin pellets treated group and HFD+orlistat treated group was $(162.62 \pm 26.15) \mathrm{pg} / \mathrm{mL}$ and $(159.67 \pm 19.13) \mathrm{pg} / \mathrm{mL}$ respectively. In the HFD+capsanthin-enriched extract pellets treated groups, there was a statistically significant $(p<0.05)$ decrease in leptin (49.03 \% lower) levels compared to the HFD control group and $19.54 \%$ reduction of leptin reported by Kim et al. (34). The same trend was noted in the HFD+capsaicin pellet (46.15\% lower) and HFD + orlistat group (47.13 \% lower). The data are shown in Fig. $2 b$.

Effect of capsanthin-enriched extract pellets on HFD-induced changes in free fatty acids levels in mice

Scientific studies have established the relationship between elevated plasma free fatty acids (FFA), also referred to as non-esterified fatty acids (NEFA), and several metabolic disorders such as insulin resistance (43). Adipose tissues are responsible for the storage and release of free fatty acids. In obesity, enlarged adipose tissue and reduction in FFA clearance are responsible for elevated plasma FFA. In addition, insulin's anti-lipolytic action inhibited by FFA causes further release of FFA into the circulation. Increasing plasma FFA level in skeletal muscle reduces insulin-stimulated glucose uptake. In the body, more than $80 \%$ of glucose uptake occurs in skeletal muscle. In the liver, the suppression of hepatic glucose production is due to FFA induced insulin resistance. Chronically, elevated plasma FFA levels lead to insulin resistance in obese diabetic and non-diabetic individuals. Studies have demonstrated that normalizing plasma FFA levels resulted in insulin-stimulated glucose uptake levels in obese individuals with diabetes (44). In obesity, impaired suppression of hepatic glucose output is caused by insulin resistance and a decrease in glucose transport (45). The action of capsanthin on FFA was previously reported in the literature by Kim et al. (34), and our study showed a similar finding.

In this study, HFD+capsanthin-enriched extract pellet treated groups showed a statistically significant $(p<0.05)$ decrease in plasma FFA levels compared to the HFD control group. The HFD groups showed higher FFA $(0.1577 \pm 0.0044) \mu \mathrm{mol} / \mathrm{mL}$ compared to ND groups $(0.0621 \pm 0.0046$ 
Please note that this is an unedited version of the manuscript that has been accepted for publication. This version will undergo copyediting and typesetting before its final form for publication. We are providing this version as a service to our readers. The published version will differ from this one as a result of linguistic and technical corrections and layout editing

$\mu \mathrm{mol} / \mathrm{mL})$. The FFA levels for low, mid, and high dose HFD+capsanthin-enriched extract pellet treated groups were $(0.0677 \pm 0.0113),(0.0717 \pm 0.007)$, and $(10.0827 \pm 0.0149) \mu \mathrm{mol} / \mathrm{mL}$ respectively, and these values are close to those of the ND groups. The FFA level was $(0.0807 \pm 0.0060) \mu \mathrm{mol} / \mathrm{mL}$ in the HFD+capsaicin pellets treated group and $(0.0851 \pm 0.0120) \mu \mathrm{mol} / \mathrm{mL}$ in the HFD+orlistat group, and these values are comparable to those of the HFD+capsanthin-enriched-extract pellets treated groups. A statistically significant $(p<0.05)$ reduction in free fatty acids was observed in the HFD+capsanthinenriched extract pellets high dose treated group and the reduction was $47.55 \%$ compared to HFD. In our study, the reduction in FFA is quite significant compared to the study reported by Kim et al. (34) where the reduction was $12.76 \%$. The reduction in FFA The data are shown in Fig. 2c.

\section{Effect of capsanthin-enriched extract pellets on HFD-induced changes in insulin levels in mice}

Kim et al. (34) showed that the supplementation of capsanthin significantly reduced insulin levels compared to the no treatment group. Similarly, in our study, capsanthin-enriched extract pellets treated groups showed a statistically significant $(p<0.05)$ reduction in insulin level compared to the HFD control group. The insulin level for the ND group and the HFD control group was $(158.77 \pm 7.53) \mathrm{pg} / \mathrm{mL}$ and (204.20 \pm 13.12$) \mathrm{pg} / \mathrm{mL}$ respectively. The insulin level in HFD+capsanthin-enriched extract pellets treated low, mid, and high dose groups was (160.02 \pm 8.90$)$, (157.81 \pm 19.39$)$, and (168.79 \pm 11.77$) \mathrm{pg} / \mathrm{mL}$ respectively. The HFD+capsaicin pellets treated group showed an insulin level of $(162.19 \pm 15.36)$ $\mathrm{pg} / \mathrm{mL}$, which is statistically significant compared to the HFD control. A non-significant change in the insulin level of $198.70 \pm 29.56) \mathrm{pg} / \mathrm{mL}$ was observed in the HFD+orlistat treated group and was almost equal to that of the HFD control. A statistically significant $(p<0.05)$ reduction in insulin was noted in the HFD+capsanthin-enriched extract pellets treated high dose group, which was $49.03 \%$ compared to HFD control group. Insulin reduction reported by Kim et al. (34) was $9.99 \%$. The data are shown in Fig. $2 d$.

\section{Gross necropsy and histopathology}

All surviving animals were subjected to necropsy and both external and internal gross pathological examinations at the end of the study period. No gross abnormality was found in the treated and control groups. One animal was found dead in the HFD+capsaicin pellet treated group during the dosing period, which was due to autolysis according to the necropsy analysis. Mice in the ND group showed normal histology without any microscopic alteration. In all treated groups, mild to moderate 
Please note that this is an unedited version of the manuscript that has been accepted for publication. This version will undergo copyediting and typesetting before its final form for publication. We are providing this version as a service to our readers. The published version will differ from this one as a result of linguistic and technical corrections and layout editing.

diffused fatty infiltration was observed in the liver. No hepatitis, hemorrhage, hepatocyte necrosis, and fat cysts were observed in the treated groups.

The histopathological scoring of the liver is presented in Table 1. Animals treated with capsanthin-enriched extract pellets and capsaicin pellets did not reveal any microscopic alteration in the liver, kidneys, and white adipose tissues (inguinal and epididymal). Histological examination revealed that the sizes of the white adipose tissues (inguinal and epididymal) were significantly smaller in the treated group, and a significant mass reduction in inguinal white adipose tissue (37.0\% lower) and epididymal white adipose tissue (43.64\% lower) was noted in the capsanthin-enriched extract pellet treated high dose group. The data are shown in Fig. 3. White adipose tissues of inguinal and epididymal of the various treated and control groups of mice are shown in Fig. 4 and Fig. 5. The larger adipocytes were observed in the HFD group compared to the ND group. In the ND group, the adipocytes were normal and the sizes were regular. The HFD control obese mice showed fat stored in adipocytes as accumulated lipid droplets that occupy most of the cytoplasm. In the HFD group, larger adipocytes were observed in the adipose tissue compared to the ND group. The normal adipocytes distribution was observed in the ND group with regular sizes of cells (Fig. 4 and Fig. 5), whereas in the HFD control, the enlarged adipocytes that occupied most of the cytoplasm were noted. However, the adipocytes of HFD+capsanthin-enriched extract pellets, HFD+capsaicin pellets, and treated groups showed a smaller size and were comparable to the HFD+orlistat and ND groups.

\section{Hematology and clinical chemistry}

Blood acts as a pathological reflector of the status of the animals exposed to phytochemicals and plays a vital role in the biological, pathological, and nutritional status of an animal (46). The examination of hematology parameters provides the opportunity to clinically investigate the impact of highly standardized capsanthin. The effect of capsanthin on the hematology parameters was not assessed and reported previously. We examined the clinical chemistry and hematology parameters and reported to the obesity biomarkers. There was no significant change in the hematology (Table S5) and clinical chemistry parameters (Table 2) in all control and treated groups. However, a non-significant reduction of the total cholesterol, LDL, HDL, triglycerides, and blood glucose levels was observed in the capsanthin-enriched extract pellets treated groups compared to the HFD control group. 
Please note that this is an unedited version of the manuscript that has been accepted for publication. This version will undergo copyediting and typesetting before its final form for publication. We are providing this version as a service to our readers. The published version will differ from this one as a result of linguistic and technical corrections and layout editing

\section{CONCLUSIONS}

In this study, we demonstrated that highly enriched and stabilized capsanthin-enriched extract pellets significantly reduced mass gain in all low, mid, and high dose groups treated and that these effects are comparable to capsaicin pellets and the reference drug orlistat. The serum, glucose, total cholesterol, LDL, and HDL triglycerides levels were lower in the capsanthin-enriched extract pellets and capsaicin pellets treated groups than in the HFD control group, whereas the obesity marker adiponectin is significantly higher in the capsanthin-enriched extract pellets and capsaicin pellets treated groups. The capsanthin-enriched extract pellets and capsaicin pellets treated groups showed a significant reduction in leptin, free fatty acids, and insulin. There was no change in the liver mass in all groups, but there was a significant reduction in adipose tissues (inguinal and epididymal white) in all the capsanthinenriched extract pellets and capsaicin pellets treated groups compared to the HFD group. The antiobesity effect of capsanthin-enriched extract pellets is remarkably significant than the previous findings. The reason may be due to the highest concentration of capsanthin in our capsanthin-enriched extract pellets. There was no change in hematology and biochemical parameters across the study groups. Therefore, the oral administration of capsanthin-enriched extract pellets and capsaicin pellets significantly reduces body mass gain, increases adiponectin, and decreases leptin, free fatty acids, and insulin levels without any adverse events at the dose tested. These results suggest that capsanthinenriched extract can be used as an alternative natural phytochemical in the development of functional foods with potential anti-obesity activities. Further gene expression studies should be conducted to better understand the mechanism of the highly enriched capsanthin.

\section{ACKNOWLEDGEMENTS}

We thank Phytosol India Pvt Ltd for providing capsaicin pellets and analytical instrumentation facility. Our special thanks to Vipragen Biosciences Pvt. Ltd, \#67b, Hootagalli industrial area, Mysore, 570 018, Karnataka, India for the facility provided to conduct the in vivo study and guidance.

\section{FUNDING}

Our sincere thanks to Mr. Sevanti Mehta, Unibar Corporation, 13615 Morgan Creek CT Houston, TX USA 77077 for financial support of the study.

\section{CONFLICT OF INTEREST}

Authors have no conflict of interest with this study. 
Please note that this is an unedited version of the manuscript that has been accepted for publication. This version will undergo copyediting and typesetting before its final form for publication. We are providing this version as a service to our readers. The published version will differ from this one as a result of linguistic and technical corrections and layout editing.

\section{SUPPLEMENTARY MATERIALS}

All supplementary materials are available at www.ftb. com.hr.

\section{AUTHORS' CONTRIBUTION}

V.S. performed the study, collected, analyzed and interpreted data and drafted the article. R.S. supervised the study, critically revised the data and interpretation, and checked and approved the final version.

ORCID ID

V. Shanmugham https://orcid.org/0000-0003-0000-0222

R. Subban https://orcid.org/0000-0002-3197-0486

\section{REFERENCES}

1. Roth J, Qiang X, Marbán SL, Redelt H, Lowell BC. The obesity pandemic: where have we been and where are we going? Obes Res Suppl 2. 2004:88S-101S. https://doi.org/10.1038/oby.2004.273

2. Hussain A, Mahawar K, Xia Z, Yang W, El-Hasani S. Obesity and mortality of COVID-19. Metaanalysis. Obes Res Clin Pract. 2020;14(4):295-300.

https://doi.org/10.1016/j.orcp.2020.07.002

3. Clemmensen C, Petersen MB, Sørensen, TIA. Will the COVID-19 pandemic worsen the obesity epidemic? Nat Rev Endocrinol.2020;16:469-470.

https://doi.org/10.1038/s41574-020-0387-z

4. Lavie CJ, Laddu D, Arena R, Ortega FB, Alpert MA, Kushner RF. Healthy weight and obesity prevention: JACC health promotion series. J Am Coll Cardiol. 2018;72(13):1506-1531. https://doi.org/10.1016/j.jacc.2018.08.1037

5. Elagizi A, Kachur S, Lavie CJ, Carbone S, Pandey A, Ortega FB, Milani RV. An overview and update on obesity and the obesity paradox in cardiovascular diseases. Prog Cardiovasc Dis. 2018;61(2):142-150. https://doi.org/10.1016/j.pcad.2018.07.003

6. Carbone S, Lavie CJ, Elagizi A, Arena R, Ventura HO. The Impact of Obesity in Heart Failure. Heart Fail Clin. 2020;16(1):71-80.

https://doi.org/10.1016/j.hfc.2019.08.008 
Please note that this is an unedited version of the manuscript that has been accepted for publication. This version will undergo copyediting and typesetting before its final form for publication. We are providing this version as a service to our readers. The published version will differ from this one as a result of linguistic and technical corrections and layout editing.

7. Neeland IJ, Yokoo T, Leinhard OD, Lavie CJ. 21st Century Advances in Multimodality Imaging of Obesity for Care of the Cardiovascular Patient. JACC Cardiovasc Imaging. 2021 Feb;14(2):482-494. https://doi.org/10.1016/j.jcmg.2020.02.031

8. Lee MJ, Wu Y, Fried SK. Adipose tissue heterogeneity: implication of depot differences in adipose tissue for obesity complications. Mol Asp Med. 2013;34(1):1-11. https://doi.org/10.1016/i.mam.2012.10.001

9. Eslami O, Khoshgoo M, Shidfar F. Dietary phytochemical index and overweight/obesity in children: a cross-sectional study. BMC Res Notes. 2020;13(1):132. https://doi.org/10.1186/s13104-020-04979-6

10. Watanabe T, Hata K, Hiwatashi K, Hori K, Suzuki N, Itoh H. Suppression of murine preadipocyte differentiation and reduction of visceral fat accumulation by a Petasites japonicus ethanol extract in mice fed a high-fat diet. Biosci Biotechnol Biochem. 2010;74(3):499-503. https://doi.org/10.1271/bbb.90684

11. Bonet ML, Canas JA, Ribot J, Palou A. Carotenoids and their conversion products in the control of adipocyte function, adiposity and obesity. Arch. Biochem. Biophys. 2015;572:112-125. https://doi.org/10.1016/j.abb.2015.02.022

12. Bonet ML, Canas JA, Ribot J, Palou A. Carotenoids in Adipose Tissue Biology and Obesity. Subcell Biochem. 2016;79:377-414. https://doi.org/10.1007/978-3-319-39126-7_15

13. Boon CS, McClements DJ, Weiss J, Decker EA. Factors influencing the chemical stability of carotenoids in foods. Crit Rev Food Sci Nutr. 2010;50(6):515-32. https://doi.org/10.1080/10408390802565889

14. Aslam S, Ahmad M, Riaz M. Stability of Carotenoids. In: Zia-Ul-Haq M, Dewanjee S, Riaz M. Carotenoids: Structure and Function in the Human Body. 2021;Springer, Cham. https://doi.org/10.1007/978-3-030-46459-2

15. Hernández-Pérez T, Gómez-García MdelR, Valverde ME, Paredes-López O. Capsicum annuum (hot pepper): an ancient Latin-American crop with outstanding bioactive compounds and nutraceutical potential. A review. Compr Rev Food Sci Food Saf. 2020;19:2972-2993. https://doi.org/10.1111/1541-4337.12634

16. Rodriguez GA. Extraction, Isolation, and Purification of Carotenoids. Curr Protoc Food Anal Chem 2001,00:F2.1.1-F2.1.8. 
Please note that this is an unedited version of the manuscript that has been accepted for publication. This version will undergo copyediting and typesetting before its final form for publication. We are providing this version as a service to our readers. The published version will differ from this one as a result of linguistic and technical corrections and layout editing.

https://doi.org/10.1002/0471142913.faf0201s00

17. Shanmugham V, Subban R. Extraction of capsanthin from Capsicum annuum $L$ fruits and its effect on carbomer-induced intraocular pressure in Albino Wistar rats. J Food Biochem. 2021;00:e13776.

https://doi.org/10.1111/jfbc.13776

18. Sinha VR, Agrawal MK, Agarwal A, Singh G, Ghai D. Extrusion-spheronization: process variables and characterization. Crit Rev Ther Drug Carrier Syst. 2009;26(3):275 331. https://doi.org/10.1615/critrevtherdrugcarriersyst.v26.i3.20

19. USP method 561. Articles of botanical origin. Rockville, MD, USA. United States Pharmacopeial (USP) Convention; 2015.

20. USP method 786. Particle size distribution. Rockville, MD, USA. United States Pharmacopeial (USP) Convention; 2015.

21. USP method 731. Loss on drying. Rockville, MD, USA. United States Pharmacopeial (USP) Convention; 2015.

22. Paprika extract, FAO JECFA 16. Prepared at the 77 JECFA, published in FAO JECFA Monographs 14 (2013), superseding tentative specifications prepared at the 69JECFA (2008). An ADI of 0-1.5 mg/kg bw was allocated at the 79 JECFA (2014). Available from: http://www.fao.org/fileadmin/user upload/jecfa additives/docs/monograph16/additive-510$\underline{\text { m16.pdf }}$

23. USP method 233. Elemental impurities. Rockville, MD, USA. United States Pharmacopeial (USP) Convention; 2015.

24. USP method 2021. Microbial Enumeration Tests. Rockville, MD, USA. United States Pharmacopeial (USP) Convention; 2015.

25. USP method 2022Absence of Specified Microorganisms. Rockville, MD, USA. United States Pharmacopeial (USP) Convention; 2015.

26. USP method 62. Microbiological examination of nonsterile products: tests for specified microorganisms. Rockville, MD, USA. United States Pharmacopeial (USP) Convention; 2015.

27. USP method 711. Dissolution. Rockville, MD, USA. United States Pharmacopeial (USP) Convention; 2015.

28. International Conference on Harmonization, Q1A(R2): Stability testing of new drug substances and products (second revision), EU: adopted by CPMP, March 2003, issued as 
Please note that this is an unedited version of the manuscript that has been accepted for publication. This version will undergo copyediting and typesetting before its final form for publication. We are providing this version as a service to our readers. The published version will differ from this one as a result of linguistic and technical corrections and layout editing

CPMP/ICH/2736/99; MHLW: adopted June 3, 2003, PFSB/ELD notification No. 0603001; FDA:

Published in the Federal Register, Vol. 68, No. 225, Friday, November 21, 2003:65717-18. http://www.ich.org/LOB/media/MEDIA419.pdf

29. Lipid bank. Data No. VCA0045. Japanese Conference on the Biochemistry of Lipids (JCBL). Available from:

http://lipidbank.jp/cgi-bin/detail.cgi?id=VCA0045.

30. Guidelines on the regulation of scientific experiments on animals. Ministry of Environment \& Forests (Animal Welfare Division) Government of India, New Delhi;June 2007. Available from: http://cpcsea.nic.in/WriteReadData/userfiles/file/SOP_CPCSEA_inner_page.pdf

31. GraphPad Prism, v.8.0, GraphPad Software Inc, San Diego, CA, USA;2018. Available from: https://www.graphpad.com/scientific-software/prism/

32. Mehta RY, Missaghi S, Tiwari SB, Rajabi-Siahboomi AR. Application of ethyl cellulose coating to hydrophilic matrices: a strategy to modulate drug release profile and reduce drug release variability. AAPS PharmSciTech. 2014;15(5):1049-59.

https://doi.org/10.1208/s12249-014-0128-5

33. Breithaupt DE, Weller P, Grashorn MA. Quantification of carotenoids in chicken plasma after feeding free or esterified lutein and capsanthin using high-performance liquid chromatography and liquid chromatography-mass spectrometry analysis. Poult Sci J. 2003;82(3):395-401. https://doi.org/10.1093/ps/82.3.395

34. Ji-Sun Kim, Tae-Youl Ha, Suna Kim, Sung-Joon Lee, Jiyun Ahn. Red paprika (Capsicum annuum L.) and its main carotenoid capsanthin ameliorate impaired lipid metabolism in the liver and adipose tissue of high-fat diet-induced obese mice. J Funct Foods. 2017;31:131-140. https://doi.org/10.1016/j.jff.2017.01.044.

35. Jo SJ, Kim JW, Choi HO, Kim JH, Kim HJ, Woo SH, Han BH. Capsanthin Inhibits both Adipogenesis in 3T3-L1 Preadipocytes and Weight Gain in High-Fat Diet-Induced Obese Mice Biomol Ther (Seoul). 2017;25(3):329-336. https://doi.org/10.4062/biomolther.2017.048

36. Wu T, Gao Y, Hao J, Geng J, Zhang J, Yin J, Liu R, Sui W, Gong L, Zhang M, Capsanthin extract prevents obesity, reduces serum TMAO levels and modulates the gut microbiota composition in high-fat-diet induced obese C57BL/6J mice, Food Res Int. 2020 Feb;128:108774. https:// doi.org/10.1016/j.foodres.2019.108774 
Please note that this is an unedited version of the manuscript that has been accepted for publication. This version will undergo copyediting and typesetting before its final form for publication. We are providing this version as a service to our readers. The published version will differ from this one as a result of linguistic and technical corrections and layout editing.

37. Fasshauer M, Bluher M. Adipokines in health and disease. Trends Pharmacol Sci. 2015;36(7): 461-70.

https://doi.org/10.1016/j.tips.2015.04.014

38. Bouskila M, Pajvani UB, Scherer PE. Adiponectin: a relevant player in PPAR gamma-agonistmediated improvements in hepatic insulin sensitivity? Int J Obes Suppl. 2005;1:S17-23. https://doi.org/10.1038/sj.ijo.0802908

39. Ronti T, Lupattelli G, Mannarino E. The endocrine function of adipose tissue: an update. Clin Endocrinol (Oxf). 2006;64(4):355-65. https://doi.org/10.1111/j.1365-2265.2006.02474.x

40. Zheng F, Zhang S, Lu W, Wu F, Yin X, Yu D, Pan Q, Li H. Regulation of insulin resistance and adiponectin signaling in adipose tissue by liver $X$ receptor activation highlights a cross-talk with PPARy. PLoS One. 2014;9(6):e101269.

https://doi.org/10.1371/journal.pone.0101269

41. Friedman J, Halaas J. Leptin and the regulation of body weight in mammals. Nature.1998; 395:763-770.

https://doi.org/10.1038/27376

42. Maffei M, Halaas J, Ravussin E, Pratley RE, Lee GH, Zhang Y, Fei H, Kim S, Lallone R, Ranganathan S. Leptin levels in human and rodent: measurement of plasma leptin and $\mathrm{Ob}$ RNA in obese and weight-reduced subjects. Nat Med. 1995;1(11):1155-1161. https://doi.org/10.1038/nm1195-1155

43. Karpe F, Dickmann JR, Frayn KN. Fatty acids, obesity, and insulin resistance: time for a reevaluation. Diabetes. 2011;60(10):2441-9.

https://doi.org/10.2337/db11-0425

44. Boden G. Obesity and free fatty acids. Endocrinol Metab Clin North Am. Sep 2008;37(3):63546.

https://doi.org/10.1016/j.ecl.2008.06.007

45. Reaven GM. Pathophysiology of insulin resistance in human disease. Physiol Rev. 1995;75(3): 473-86.

https://doi.org/10.1152/physrev.1995.75.3.473

46. Etim N. Hematological Parameters and Factors Affecting Their Values. Agricultural Science. (2014);2:37-47. https://doi.org/10.12735/as.v2i1p37. 
Please note that this is an unedited version of the manuscript that has been accepted for publication. This version will undergo copyediting and typesetting before its final form for publication. We are providing this version as a service to our readers. The published version will differ from this one as a result of linguistic and technical corrections and layout editing.

Table 1. Histopathological scoring of liver

\begin{tabular}{lccccccc}
\hline \multicolumn{1}{c}{ Observation } & \multicolumn{7}{c}{ Groups } \\
\cline { 2 - 8 } & G1 & G2 & G3 & G4 & G5 & G6 & G7 \\
\hline Fatty infiltration & - & +++ & + & + & ++ & ++ & ++ \\
Hypertrophy of adipocytes & - & +++ & + & + & ++ & ++ & ++ \\
Inguinal white adipose tissue & & & & & & & \\
Hypertrophy of adipocytes & - & +++ & + & + & ++ & ++ & ++ \\
Epididymal white adipose tissue & & & & & & & \\
Hepatitis & - & ++ & - & - & - & - & - \\
Hemorrhage & - & - & - & - & - & - & - \\
Hepatocyte necrosis & - & - & - & - & - & - & - \\
Fat cysts & - & - & - & - & - & - & -
\end{tabular}

aThe severity was evaluated based on the following scoring scheme: - =normal, +=mild effect, $++=$ moderate effect, $+++=$ severe effect. Values are expressed as mean \pm standard deviation $(N=10)$. alndicate statistically significant differences $(p<0.05)$ with respect to $G 2$. G1=normal control, G2=HFD control, G3=HFD+capsanthin enriched extract pellets (low dose), G4=HFD+capsanthin enriched extract pellets (mid dose), G5=HFD+capsanthin enriched extract pellets (high dose), G6=HFD+capsaicin pellets, G7= HFD+orlistat, HFD=high fat diet

Table 2. Clinical chemistry parameters

\begin{tabular}{cccccccc}
\hline & \multicolumn{7}{c}{ Clinical chemistry parameters } \\
\cline { 2 - 8 } Parameter & \multicolumn{7}{c}{ Groups } \\
\hline & G1 & G2 & G3 & G4 & G5 & G6 & G7 \\
\hline $\mathrm{GLU} /(\mathrm{mg} / \mathrm{dL})$ & $180.22 \pm 22.20$ & $217.07 \pm 23.57$ & $169.49 \pm 23.14$ & $206.00 \pm 62.43$ & $205.54 \pm 40.54$ & $197.68 \pm 25.13$ & $215.32 \pm 68.60$ \\
$\mathrm{CHO} /(\mathrm{mg} / \mathrm{dL})$ & $(70.92 \pm 7.80)^{\mathrm{a}}$ & $124.64 \pm 9.92$ & $114.38 \pm 27.26$ & $131.31 \pm 40.54$ & $115.44 \pm 17.63$ & $118.16 \pm 20.50$ & $120.30 \pm 25.27$ \\
$\mathrm{TRI} /(\mathrm{mg} / \mathrm{dL})$ & $48.55 \pm 15.38$ & $63.52 \pm 21.56$ & $70.41 \pm 16.73$ & $81.11 \pm 24.75$ & $94.72 \pm 39.46$ & $84.81 \pm 22.14$ & $75.66 \pm 25.24$ \\
$\mathrm{HDL} /(\mathrm{mg} / \mathrm{dL})$ & $(38.53 \pm 5.35)^{\mathrm{a}}$ & $63.29 \pm 3.42$ & $55.95 \pm 12.61$ & $62.83 \pm 19.45$ & $58.96 \pm 11.02$ & $60.52 \pm 10.65$ & $54.58 \pm 18.13$ \\
$\mathrm{LDL} /(\mathrm{mg} / \mathrm{dL})$ & $3.03 \pm 0.37$ & $4.54 \pm 0.72$ & $5.48 \pm 3.11$ & $4.40 \pm 1.94$ & $4.71 \pm 0.70$ & $4.55 \pm 1.09$ & $6.63 \pm 4.86$ \\
$\mathrm{URE} /(\mathrm{mg} / \mathrm{dL})$ & $43.20 \pm 6.33$ & $48.31 \pm 7.84$ & $50.59 \pm 5.52$ & $52.85 \pm 3.45$ & $50.45 \pm 5.59$ & $55.08 \pm 7.43$ & $53.27 \pm 5.21$ \\
$\mathrm{CRE} /(\mathrm{mg} / \mathrm{dL})$ & $0.38 \pm 0.03$ & $0.38 \pm 0.02$ & $0.36 \pm 0.02$ & $0.37 \pm 0.02$ & $0.39 \pm 0.02$ & $0.39 \pm 0.02$ & $0.40 \pm 0.01$ \\
$\mathrm{AST} /(\mathrm{U} / \mathrm{L})$ & $71.10 \pm 21.80$ & $132.19 \pm 71.83$ & $88.09 \pm 36.86$ & $176.49 \pm 89.18$ & $92.47 \pm 39.52$ & $110.31 \pm 51.49$ & $126.49 \pm 62.17$ \\
$\mathrm{ALT} / \mathrm{U} / \mathrm{L})$ & $33.55 \pm 1.60$ & $43.66 \pm 12.39$ & $31.14 \pm 13.00$ & $48.22 \pm 11.03$ & $32.86 \pm 5.11$ & $33.21 \pm 6.25$ & $35.98 \pm 13.99$
\end{tabular}

Values are expressed as mean \pm standard deviation $(N=10)$. alndicate statistically significant differences $(p<0.05)$ with respect to G2. G1=normal control, G2=HFD control, G3=HFD+capsanthin enriched extract pellets (low dose), G4=HFD+capsanthin enriched extract pellets (mid dose), G5=HFD+capsanthin enriched extract pellets (high dose), G6=HFD+capsaicin pellets, G7= HFD+orlistat, HFD=high fat diet, S.D.=standard deviation, Glu=glucose, $\mathrm{CHO}=$ cholesterol, $\mathrm{TRIG}=$ triglycerides, $\mathrm{HDL}=$ high density lipoprotein, $\mathrm{LDL}=$ low density lipoprotein, URE=urea, $\mathrm{CRT}=$ creatinine, $\mathrm{AST}=$ aspartate aminotransferase, $\mathrm{ALT}=$ alanine aminotransferase, $\mathrm{TP}=$ total protein, $\mathrm{ALB}=$ albumin, $\mathrm{ALP}=$ alkaline phosphatase 
Please note that this is an unedited version of the manuscript that has been accepted for publication. This version will undergo copyediting and typesetting before its final form for publication. We are providing this version as a service to our readers. The published version will differ from this one as a result of linguistic and technical corrections and layout editing.

a)

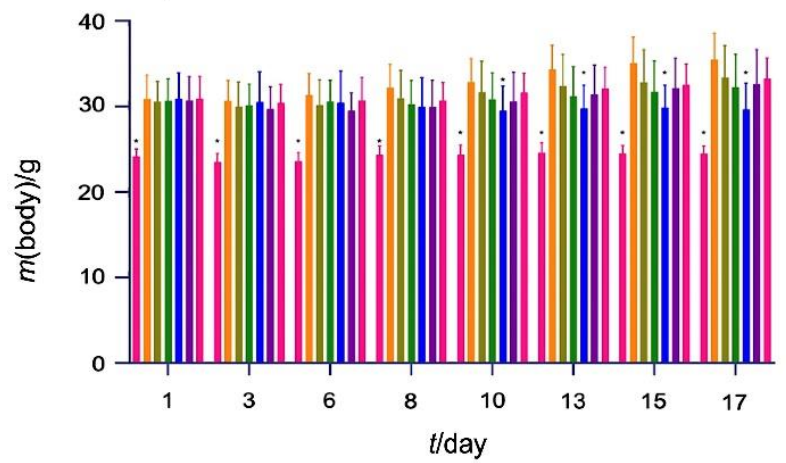

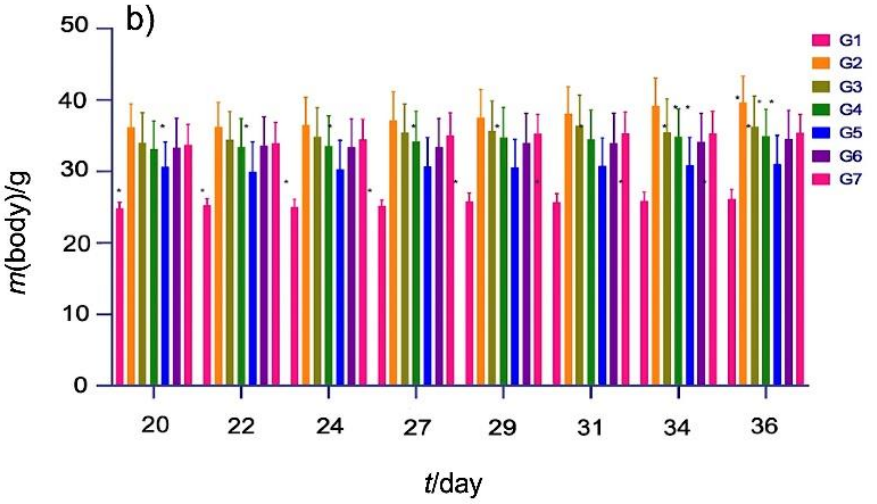

Fig. 1. Comparison of body mass in control and treated groups $N=10$. a) body mass days 1 to 7 , and b) body mass days 20 to 36 . Values are expressed in mean \pm standard deviation. * indicate statistically significant differences $(p<0.05)$ with respect to G2. G1=normal control, G2=HFD control, G3=HFD+capsanthin enriched extract pellets (low dose), G4=HFD+capsanthin enriched extract pellets (mid dose), G5=HFD+capsanthin enriched extract pellets (high dose), G6=HFD+capsaicin pellets, G7=HFD+orlistat. HFD=high fat diet. 
Please note that this is an unedited version of the manuscript that has been accepted for publication. This version will undergo copyediting and typesetting before its final form for publication. We are providing this version as a service to our readers. The published version will differ from this one as a result of linguistic and technical

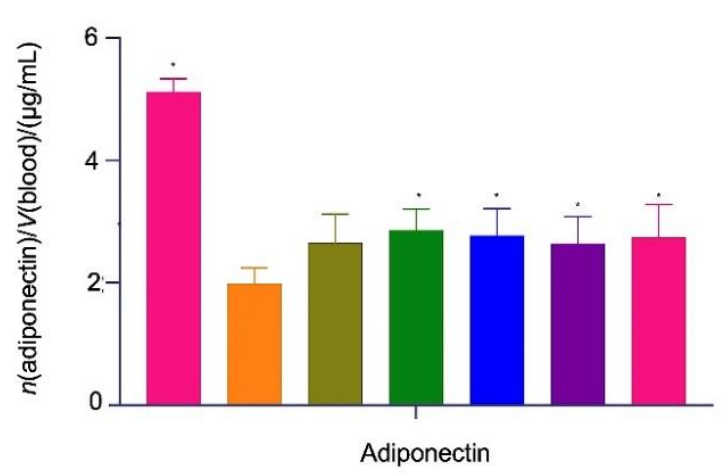

c)

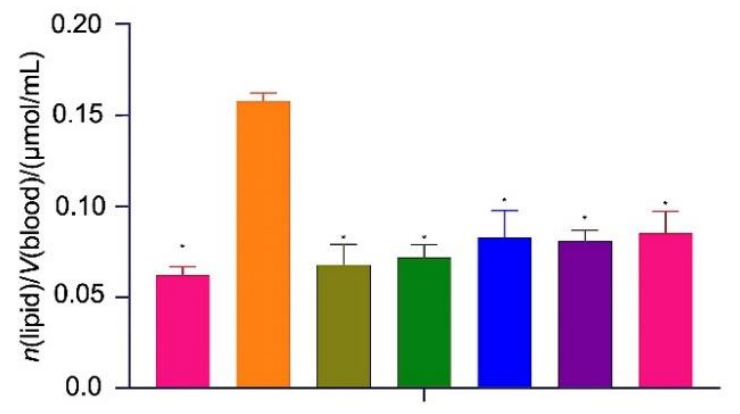

Free fatty acids

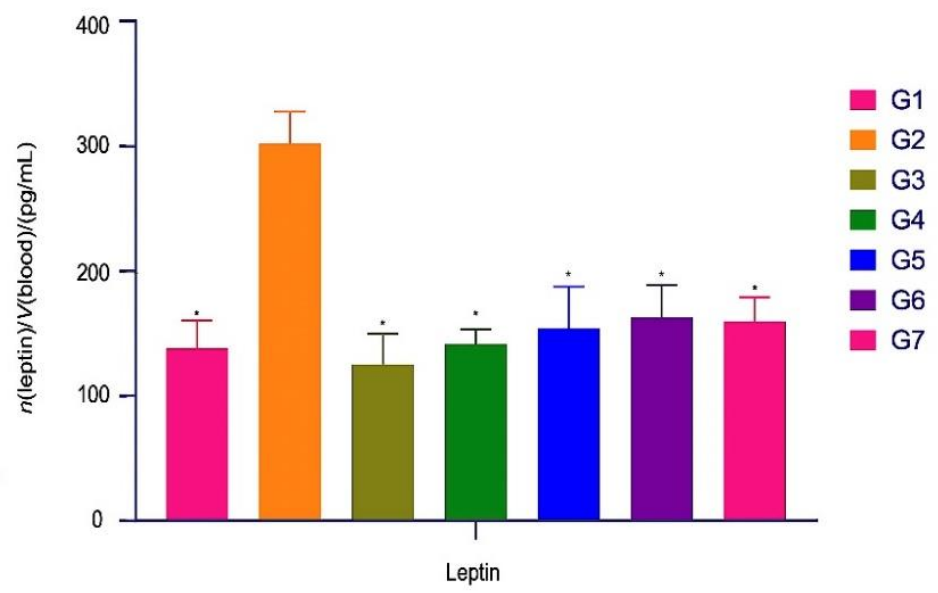

d)

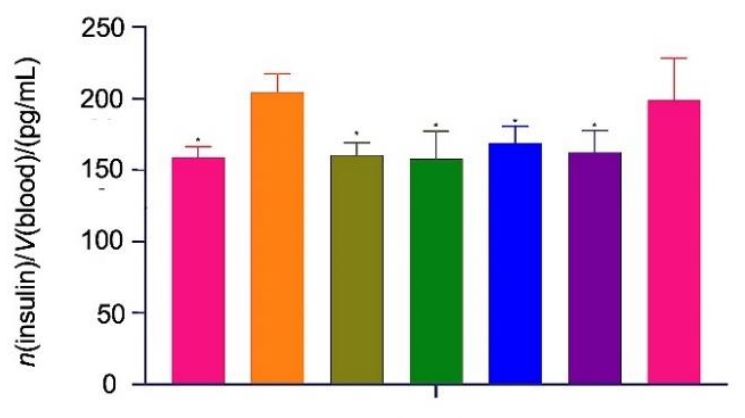

Insulin

Fig 2. Effect of capsanthin on obesity biomarkers-adiponectin, leptin, free fatty acids and Insulin $N=10$. a) insulin, b) leptin c), adiponectin, and d) free fatty acids. G1=normal control, G2=HFD control, G3=HFD+capsanthin enriched extract pellets (low dose), G4=HFD+capsanthin enriched extract pellets (mid dose), G5=HFD+ capsanthin enriched extract pellets (high dose), G6=HFD+capsaicin pellets, G7= HFD+orlistat. HFD=high fat diet. Values are expressed in mean \pm standard deviation. indicate statistically significant differences $(p<0.05)$ with respect to G2. 
Please note that this is an unedited version of the manuscript that has been accepted for publication. This version will undergo copyediting and typesetting before its final form for publication. We are providing this version as a service to our readers. The published version will differ from this one as a result of linguistic and technical corrections and layout editing.

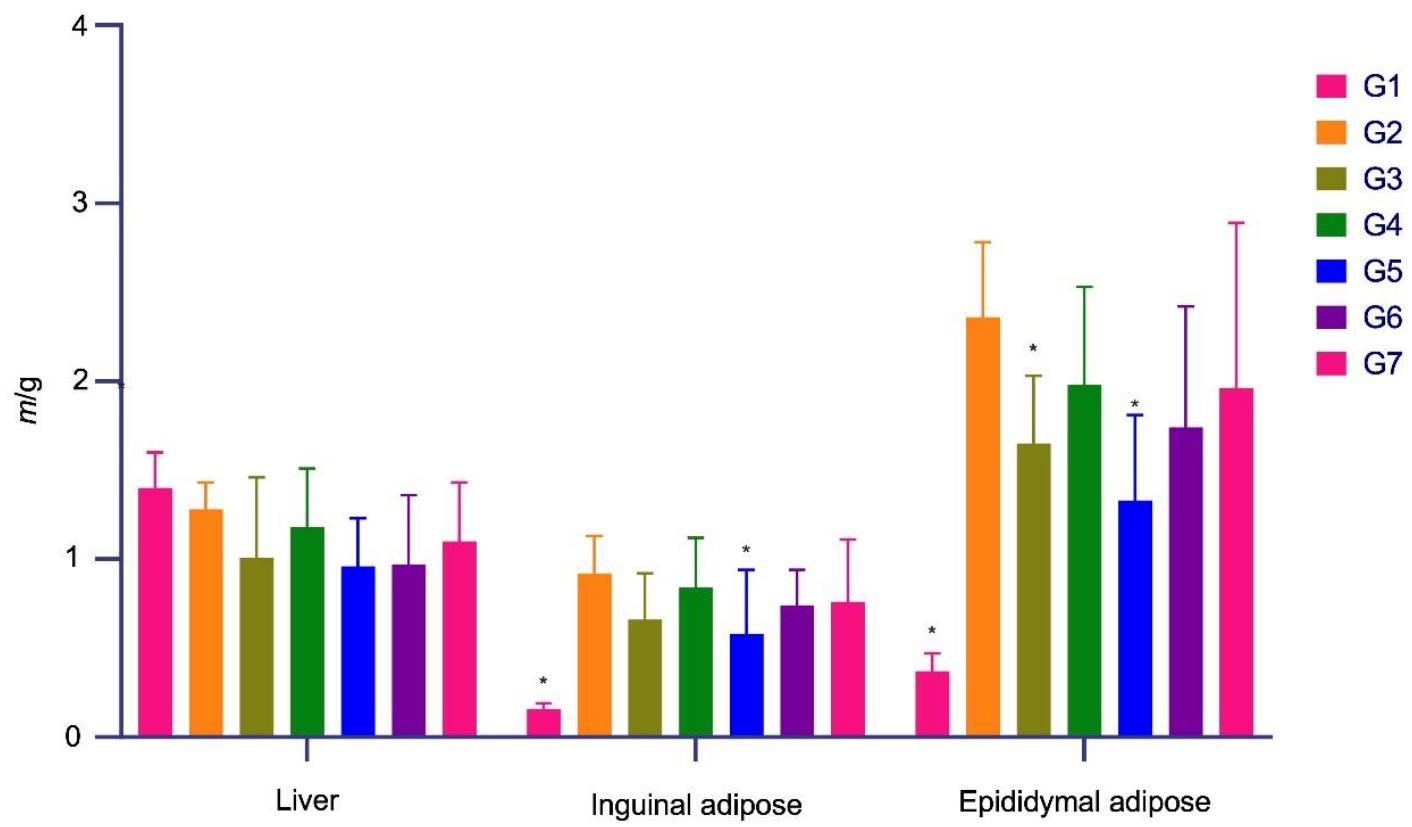

Fig 3. Comparison of liver, inguinal white adipose and epididymal fat pad mass $N=10$. Values are expressed in mean \pm standard deviation. *indicate statistically significant differences $(p<0.05)$ with respect to $G 2$. G1=Normal control, G2=HFD control, G3=HFD+ Capsanthin enriched extract pellets (Low dose), G4=HFD+ capsanthin enriched extract pellets (mid dose), G5=HFD+ capsanthin enriched extract pellets (high dose), G6=HFD+capsaicin pellets, G7= HFD+orlistat. HFD=high fat diet. 
Please note that this is an unedited version of the manuscript that has been accepted for publication. This version will undergo copyediting and typesetting before its final form for publication. We are providing this version as a service to our readers. The published version will differ from this one as a result of linguistic and technical corrections and layout editing.
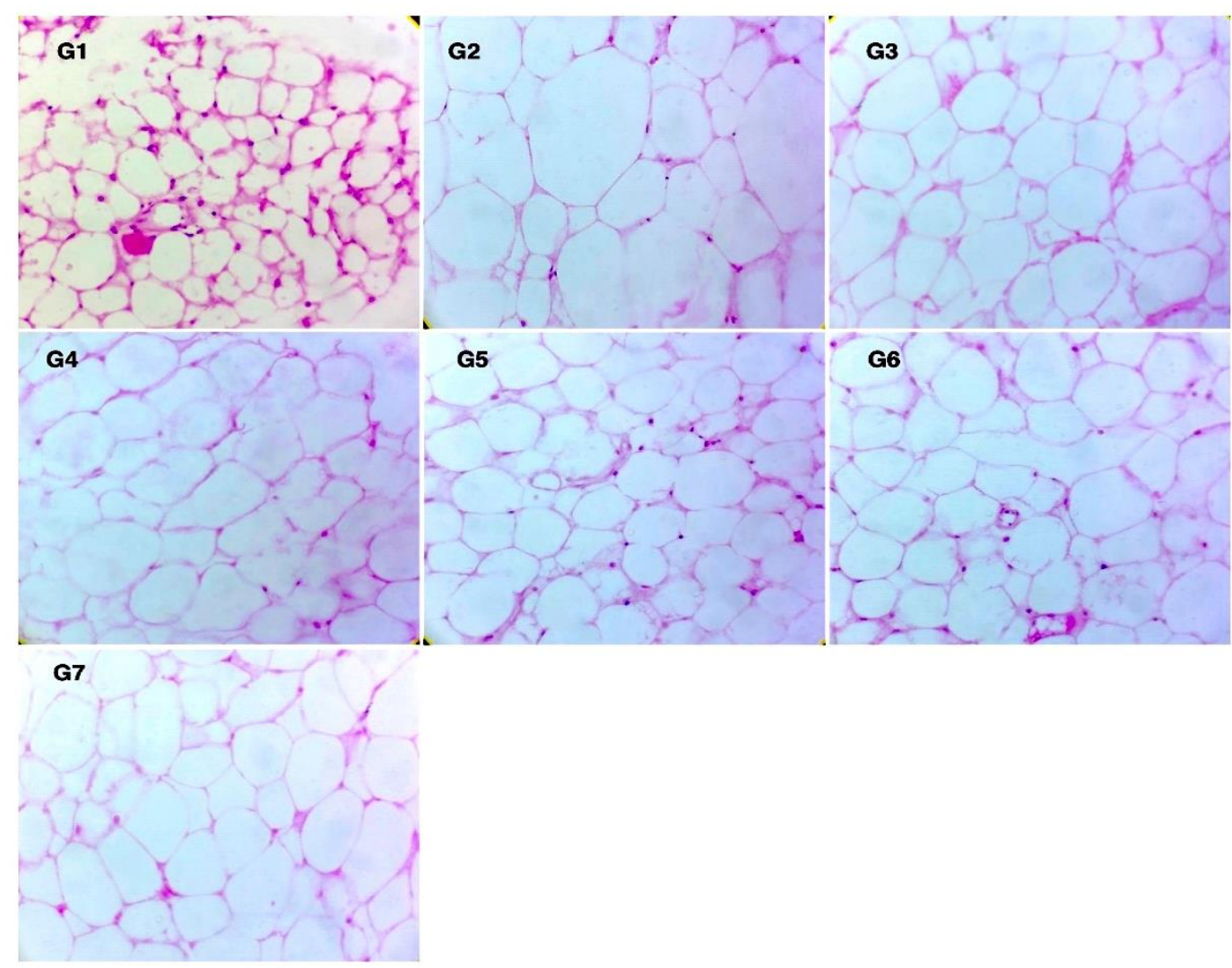

Fig 4. Photomicrographs of inguinal white adipose tissue (Hematoxylin \& Eosin, 40x) Smaller size of adipocytes was clearly observed in G1 (normal control). The enlarged adipocytes observed in G2 (HFD control). Reduction in the size of adipocytes observed in all treated groups (G3 to G7). G1=Normal control, G2=HFD control, G3=HFD+ Capsanthin enriched extract pellets (Low dose), G4=HFD+ capsanthin enriched extract pellets (mid dose), G5=HFD+ capsanthin enriched extract pellets (high dose), G6=HFD+capsaicin pellets, G7= HFD+orlistat. HFD=high fat diet. 

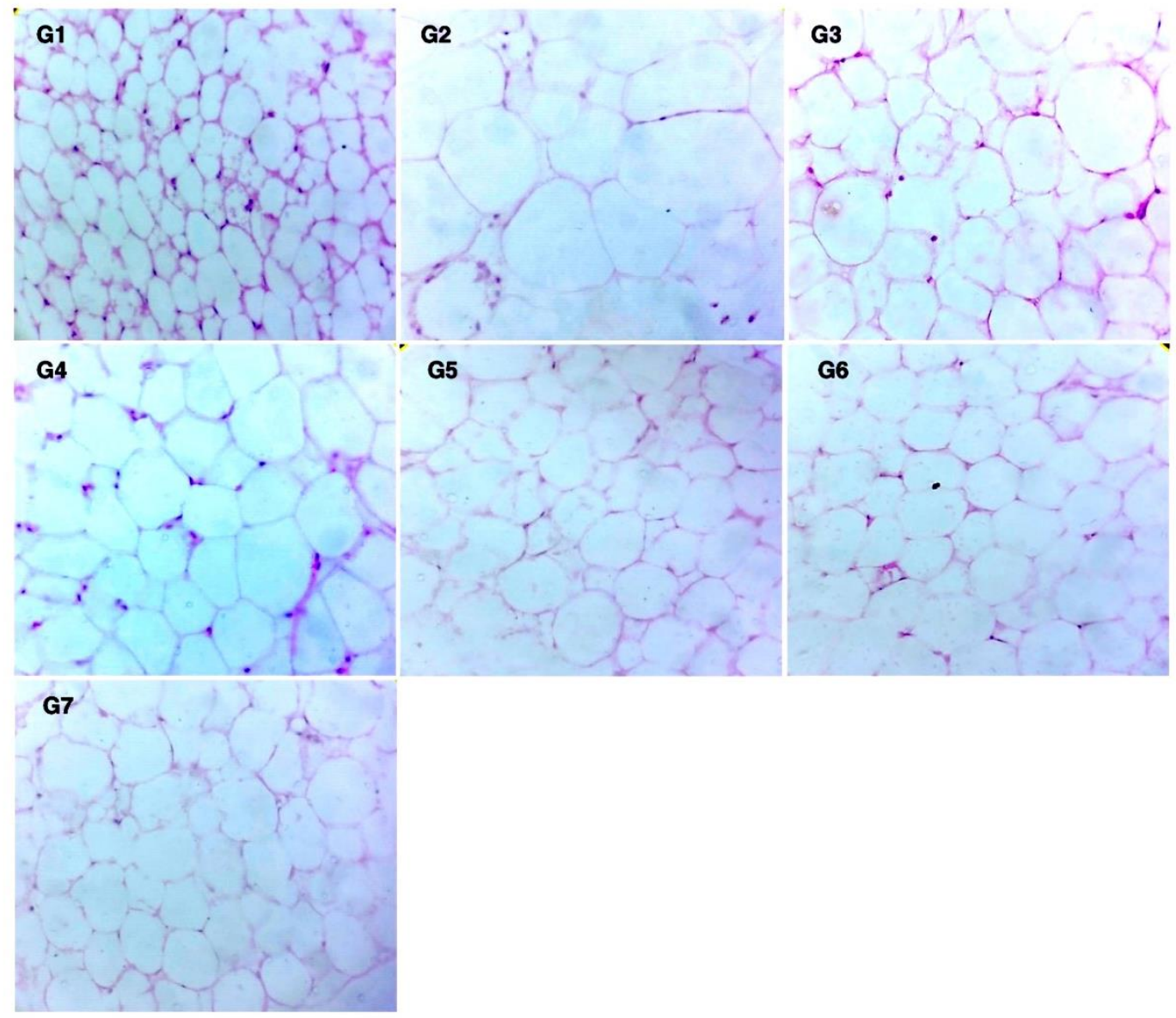

Fig 5. Photomicrographs of epididymal white adipose tissue (Hematoxylin \& Eosin, 40x) Smaller size of adipocytes was clearly observed in G1 (normal control). The enlarged adipocytes observed in G2 (HFD control). Reduction in the size of adipocytes observed in all treated groups (G3 to G7). G1=Normal control, G2=HFD control, G3=HFD+ Capsanthin enriched extract pellets (Low dose), G4=HFD+ capsanthin enriched extract pellets (mid dose), G5=HFD+ capsanthin enriched extract pellets (high dose), G6=HFD+capsaicin pellets, G7= HFD+orlistat. HFD=high fat diet. 
Please note that this is an unedited version of the manuscript that has been accepted for publication. This version will undergo copyediting and typesetting before its final form for publication. We are providing this version as a service to our readers. The published version will differ from this one as a result of linguistic and technical corrections and layout editing.

\section{Supplementary Tables and Figures}

Table S1. Composition of capsanthin-enriched extract pellets

\begin{tabular}{ccc}
\hline Matrix & Ingredient & $\mathrm{m} / \mathrm{g}$ \\
\hline Core & capsanthin-enriched extract crystals & 1000 \\
& pharma grade sucrose & 200 \\
& microcrystalline cellulose & 200 \\
& polyvinyl pyrrolidine K 30 5\% & QS \\
in isopropyl alcohol & \\
Coating & ethyl cellulose N 20 & \\
& sodium alginate & 20 \\
& ethanol & 20 \\
& water & QS \\
QS=quantity sufficient & &
\end{tabular}

Table S2. Specification of capsanthin-enriched extract pellets

\begin{tabular}{|c|c|c|}
\hline Parameter & Specification & Method \\
\hline \multicolumn{3}{|l|}{ Physical } \\
\hline Description & dark red pellets & \multirow{2}{*}{$\begin{array}{r}\text { Organoleptic } \\
\text { (22) }\end{array}$} \\
\hline Identification & to comply by UV & \\
\hline \multirow[t]{2}{*}{ Solubility } & slightly soluble in alcohol & \multirow[b]{2}{*}{ (19) } \\
\hline & and insoluble in water & \\
\hline Particle size & $90 \%$ passes through 30 mesh & (20) \\
\hline Loss on drying & $<2.0 \%$ & (21) \\
\hline \multicolumn{3}{|l|}{ Chemical } \\
\hline \multicolumn{3}{|l|}{ Assay } \\
\hline Capsanthin & $w>50.0 \%$ & (22) \\
\hline Carotenoids & $w>60.0 \%$ & (22) \\
\hline \multicolumn{3}{|l|}{ Elemental impurities } \\
\hline Lead & $<5.0 \mathrm{mg} / \mathrm{kg}$ & (23) \\
\hline Arsenic & $\mathrm{hg} / \mathrm{kg}$ & (23) \\
\hline Cadmium & $<1.0 \mathrm{mg} / \mathrm{kg}$ & (23) \\
\hline Mercury & $<1.0 \mathrm{mg} / \mathrm{kg}$ & (23) \\
\hline \multicolumn{3}{|l|}{ Microbiological profile } \\
\hline Total aerobic microbial count & $<3000 \mathrm{CFU} / \mathrm{g}$ & (24) \\
\hline Total yeast and mold count & $<100 \mathrm{CFU} / \mathrm{g}$ & (24) \\
\hline \multicolumn{3}{|c|}{ Escherichia coli $\quad$ negative in $10 \mathrm{~g}$ of sample } \\
\hline Salmonella species & negative in $10 \mathrm{~g}$ of sample & (25) \\
\hline \multicolumn{3}{|c|}{ Staphylococcus aureus negative in $10 \mathrm{~g}$ of sample } \\
\hline Pseudomonas aeruginosa & negative in $10 \mathrm{~g}$ of sample & (26) \\
\hline
\end{tabular}


Please note that this is an unedited version of the manuscript that has been accepted for publication. This version will undergo copyediting and typesetting before its final form for publication. We are providing this version as a service to our readers. The published version will differ from this one as a result of linguistic and technical corrections and layout editing.

Table S3. Capsanthin-enriched extract room temperature stability data

\begin{tabular}{llllll}
\hline Parameter & Specification & \multicolumn{3}{c}{ t/month } \\
\cline { 3 - 6 } & & 0 & 3 & 6 & 12 \\
\hline Description & Dark red & Dark red & Same as & Same as & Same as \\
pellets & pellets & initial & initial & initial \\
Identification & $\begin{array}{l}\text { To comply } \\
\text { by UV }\end{array}$ & complies & complies & complies & complies \\
Loss on drying/\% & 1.24 & 1.32 & 1.88 & 1.52 & 1.79 \\
w/capsanthin/\% & 54.21 & 54.19 & 54.09 & 53.88 & 53.97 \\
w/carotenoids/\% & 62.86 & 62.99 & 61.79 & 62.16 & 62.58 \\
Change of colour & - & No & No & No & No \\
\hline
\end{tabular}

Storage conditions: temperature $=(25 \pm 2) \stackrel{\circ}{\circ}$, relative humidity $=(60 \pm 5) \%$

Table S4. Summary of feed consumption

Food consumption/(g/day)

\begin{tabular}{lccccccc}
\cline { 2 - 7 }$t /$ day & $\mathrm{G} 1$ & $\mathrm{G} 2$ & $\mathrm{G} 3$ & $\mathrm{G} 4$ & $\mathrm{G} 5$ & $\mathrm{G} 6$ & $\mathrm{G} 7$ \\
\hline $1-3$ & $(4.16 \pm 0.46)^{\mathrm{a}}$ & $2.69 \pm 0.25$ & $2.44 \pm 0.37$ & $2.47 \pm 0.33$ & $2.50 \pm 0.51$ & $2.48 \pm 0.68$ & $2.73 \pm 0.34$ \\
$3-6$ & $(4.02 \pm 0.46)^{\mathrm{a}}$ & $2.78 \pm 0.21$ & $2.58 \pm 0.33$ & $2.69 \pm 0.30$ & $2.54 \pm 0.60$ & $2.33 \pm 0.46$ & $2.75 \pm 0.34$ \\
$6-8$ & $(3.94 \pm 0.38)^{\mathrm{a}}$ & $3.01 \pm 0.40$ & $2.78 \pm 0.65$ & $(2.16 \pm 0.75)^{\mathrm{a}}$ & $(2.30 \pm 0.68)^{\mathrm{a}}$ & $2.93 \pm 0.70$ & $2.57 \pm 0.37$ \\
810 & $(4.04 \pm 0.43)^{\mathrm{a}}$ & $2.89 \pm 0.48$ & $2.74 \pm 0.38$ & $2.51 \pm 0.45$ & $2.66 \pm 0.31$ & $3.01 \pm 0.64$ & $2.84 \pm 0.35$ \\
$10-13$ & $(3.75 \pm 0.18)^{\mathrm{a}}$ & $2.89 \pm 0.26$ & $2.78 \pm 0.25$ & $2.66 \pm 0.48$ & $(2.46 \pm 0.40)^{\mathrm{a}}$ & $2.86 \pm 0.35$ & $2.64 \pm 0.40$ \\
$13-15$ & $(3.74 \pm 0.61)^{\mathrm{a}}$ & $2.97 \pm 0.30$ & $2.71 \pm 0.27$ & $2.59 \pm 0.50$ & $2.43 \pm 0.64$ & $3.07 \pm 0.59$ & $2.70 \pm 0.56$ \\
$15-17$ & $(3.69 \pm 0.25)^{\mathrm{a}}$ & $2.87 \pm 0.27$ & $2.58 \pm 0.27$ & $2.65 \pm 0.44$ & $2.48 \pm 0.37$ & $2.74 \pm 0.60$ & $2.87 \pm 0.35$ \\
$17-20$ & $(4.05 \pm 0.50)^{\mathrm{a}}$ & $2.85 \pm 0.47$ & $2.74 \pm 0.17$ & $2.91 \pm 0.47$ & $2.71 \pm 0.22$ & $2.72 \pm 0.60$ & $2.84 \pm 0.50$ \\
$20-22$ & $(4.85 \pm 0.34)^{\mathrm{a}}$ & $3.03 \pm 1.08$ & $2.94 \pm 0.75$ & $3.31 \pm 1.43$ & $2.67 \pm 0.76$ & $2.93 \pm 0.86$ & $2.81 \pm 0.82$ \\
$22-24$ & $(4.14 \pm 0.53)^{\mathrm{a}}$ & $2.80 \pm 0.45$ & $3.00 \pm 0.35$ & $2.94 \pm 0.30$ & $2.85 \pm 0.36$ & $3.08 \pm 0.32$ & $3.03 \pm 0.26$ \\
$24-27$ & $(4.34 \pm 0.49)^{\mathrm{a}}$ & $2.90 \pm 0.38$ & $2.88 \pm 0.25$ & $2.96 \pm 0.25$ & $2.84 \pm 0.24$ & $2.98 \pm 0.21$ & $3.11 \pm 0.33$ \\
$27-29$ & $(4.94 \pm 0.34)^{\mathrm{a}}$ & $3.01 \pm 0.28$ & $3.01 \pm 0.30$ & $3.01 \pm 0.37$ & $2.87 \pm 0.41$ & $3.04 \pm 0.17$ & $3.11 \pm 0.22$ \\
$29-31$ & $(4.32 \pm 0.47)^{\mathrm{a}}$ & $2.97 \pm 0.22$ & $2.82 \pm 0.38$ & $3.04 \pm 0.72$ & $2.76 \pm 0.38$ & $3.04 \pm 0.29$ & $3.07 \pm 0.18$ \\
$31-34$ & $(4.11 \pm 0.32)^{\mathrm{a}}$ & $3.03 \pm 0.31$ & $2.90 \pm 0.32$ & $2.92 \pm 0.29$ & $2.95 \pm 0.29$ & $3.29 \pm 0.69$ & $3.10 \pm 0.27$ \\
$34-36$ & $(4.17 \pm 0.36)^{\mathrm{a}}$ & $3.14 \pm 0.39$ & $3.08 \pm 0.23$ & $3.24 \pm 0.31$ & $3.03 \pm 0.33$ & $3.07 \pm 0.30$ & $3.24 \pm 0.24$ \\
\hline
\end{tabular}

Values are expressed as mean \pm standard deviation $(N=10)$. alndicate statistically significant differences $(p<0.05)$ with respect to G2. G1=normal control, G2=HFD control, G3=HFD+capsanthin enriched extract pellets (low dose), G4=HFD+capsanthin enriched extract pellets (mid dose), G5=HFD+capsanthin enriched extract pellets (high dose), G6=HFD+capsaicin pellets, G7= HFD+orlistat, HFD=high fat diet 
Please note that this is an unedited version of the manuscript that has been accepted for publication. This version will undergo copyediting and typesetting before its final form for publication. We are providing this version as a service to our readers. The published version will differ from this one as a result of linguistic and technical corrections and layout editing.

\section{Table S5. Hematology parameters}

\begin{tabular}{|c|c|c|c|c|c|c|c|}
\hline \multirow[b]{3}{*}{ Parameter } & \multicolumn{7}{|c|}{ Hematological parameters } \\
\hline & \multicolumn{7}{|c|}{ Groups } \\
\hline & G1 & G2 & G3 & G4 & G5 & G6 & G7 \\
\hline $\mathrm{WBC} /\left(10^{3} / \mathrm{mm}^{3}\right)$ & $5.78 \pm 1.10$ & $3.82 \pm 1.11$ & $4.62 \pm 1.10$ & $4.70 \pm 1.48$ & $4.60 \pm 0.79$ & $3.68 \pm 0.82$ & $4.60 \pm 0.29$ \\
\hline $\mathrm{RBC} /\left(10^{6} / \mathrm{mm}^{3}\right)$ & $10.69 \pm 1.45$ & $12.69 \pm 0.71$ & $12.65 \pm 1.50$ & $12.15 \pm 0.50$ & $11.44 \pm 1.70$ & $(10.66 \pm 0.80)^{a}$ & $10.76 \pm 0.96$ \\
\hline $\mathrm{HGB} /(\mathrm{g} / \mathrm{dL})$ & $(14.36 \pm 187)^{\mathrm{a}}$ & $17.34 \pm 0.63$ & $16.10 \pm 2.11$ & $16.00 \pm 0.45$ & $15.38 \pm 2.21$ & $(14.60 \pm 1.02)^{a}$ & $(14.52 \pm 1.50)^{\mathrm{a}}$ \\
\hline $\mathrm{HCT} / \%$ & $52.26 \pm 7.34$ & $61.44 \pm 3.17$ & $61.08 \pm 7.27$ & $58.84 \pm 2.30$ & $54.72 \pm 8.18$ & $51.56 \pm 4.03$ & $51.74 \pm 5.23$ \\
\hline $\mathrm{PLT} /\left(10^{3} / \mathrm{mm}^{3}\right)$ & $1377.20 \pm 286.23$ & $767.80 \pm 145.47$ & $1165.33 \pm 233.87$ & $1224.40 \pm 265.93$ & $1500.75 \pm 114.11$ & $1464.25 \pm 188.35$ & $1550.67 \pm 15.31$ \\
\hline $\mathrm{MCV} / \mu \mathrm{m}^{3}$ & $49.00 \pm 0.00$ & $48.40 \pm 0.55$ & $48.20 \pm 0.45$ & $48.40 \pm 0.55$ & $47.80 \pm 0.45$ & $48.20 \pm 0.45$ & $48.20 \pm 0.84$ \\
\hline $\mathrm{MCH} / \mathrm{pg}$ & $13.42 \pm 0.20$ & $13.66 \pm 0.30$ & $(12.72 \pm 0.52)^{a}$ & $13.18 \pm 0.19$ & $13.46 \pm 0.15$ & $13.72 \pm 0.19$ & $13.48 \pm 0.28$ \\
\hline $\mathrm{MCHC} /(\mathrm{g} / \mathrm{dL})$ & $27.46 \pm 0.40$ & $28.22 \pm 0.51$ & $(26.34 \pm 0.92)^{a}$ & $(27.22 \pm 0.43)^{a}$ & $28.12 \pm 0.31$ & $28.38 \pm 0.37$ & $28.02 \pm 0.29$ \\
\hline NEU/\% & $23.60 \pm 2.19$ & $26.00 \pm 4.00$ & $27.60 \pm 3.58$ & $24.00 \pm 2.83$ & $26.00 \pm 2.83$ & $28.80 \pm 3.63$ & $25.60 \pm 3.29$ \\
\hline LYM/\% & $70.40 \pm 2.61$ & $69.60 \pm 3.29$ & $69.60 \pm 1.67$ & $72.00 \pm 2.00$ & $70.00 \pm 1.41$ & $67.60 \pm 2.19$ & $69.20 \pm 1.10$ \\
\hline EOS/\% & $2.80 \pm 0.84$ & $2.20 \pm 0.84$ & $1.20 \pm 1.10$ & $1.40 \pm 1.14$ & $3.00 \pm 1.87$ & $1.80 \pm 0.84$ & $2.20 \pm 1.30$ \\
\hline MON/\% & $3.20 \pm 0.84$ & $2.20 \pm 0.84$ & $1.60 \pm 1.34$ & $2.60 \pm 1.14$ & $3.00 \pm 1.87$ & $1.80 \pm 1.30$ & $3.00 \pm 1.87$ \\
\hline BAS/\% & $0.00 \pm 0.00$ & $0.00 \pm 0.00$ & $0.00 \pm 0.00$ & $0.00 \pm 0.00$ & $0.00 \pm 0.00$ & $0.00 \pm 0.00$ & $0.00 \pm 0.00$ \\
\hline
\end{tabular}

Values are expressed as mean \pm standard deviation $(N=10)$. alndicate statistically significant differences $(\mathrm{p}<0.05)$ with respect to $\mathrm{G} 2$. $\mathrm{G} 1=$ normal control, G2=HFD control, G3=HFD+capsanthin enriched extract pellets (low dose), G4=HFD+capsanthin enriched extract pellets (mid dose), G5=HFD+capsanthin enriched extract pellets (high dose), G6=HFD+capsaicin pellets, G7= HFD+orlistat. HFD=high fat diet. WBC=total leukocyte count, RBC=erythrocyte count, $\mathrm{HGB}=$ hemoglobin, $\mathrm{HCT}=$ hematocrite, $\mathrm{PLT}=$ platelet, $\mathrm{MCV}=$ mean corpuscular volume, $\mathrm{MCH}=$ mean corpuscular haemoglobin, $\mathrm{MCHC}=\mathrm{mean}$ corpuscular hemoglobin concentration, NEU=neutrophils, LYM=lymphocytes, EOS=eosinophils, MON=monocytes, BAS=basophils 
Please note that this is an unedited version of the manuscript that has been accepted for publication. This version will undergo copyediting and typesetting before its final form for publication. We are providing this version as a service to our readers. The published version will differ from this one as a result of linguistic and technical corrections and layout editing.
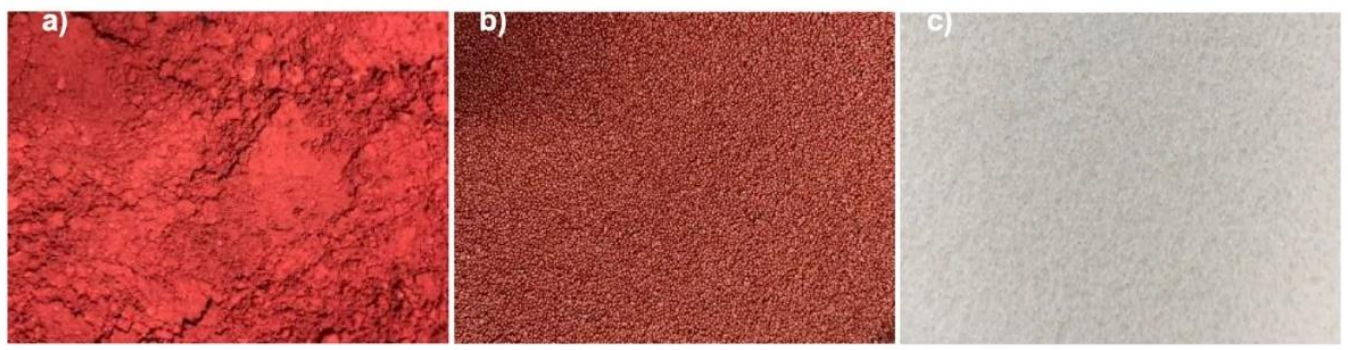

Fig. S1. a) capsanthin enriched extract crystals, b) capsanthin enriched extract pellets, and c) capsaicin pellets

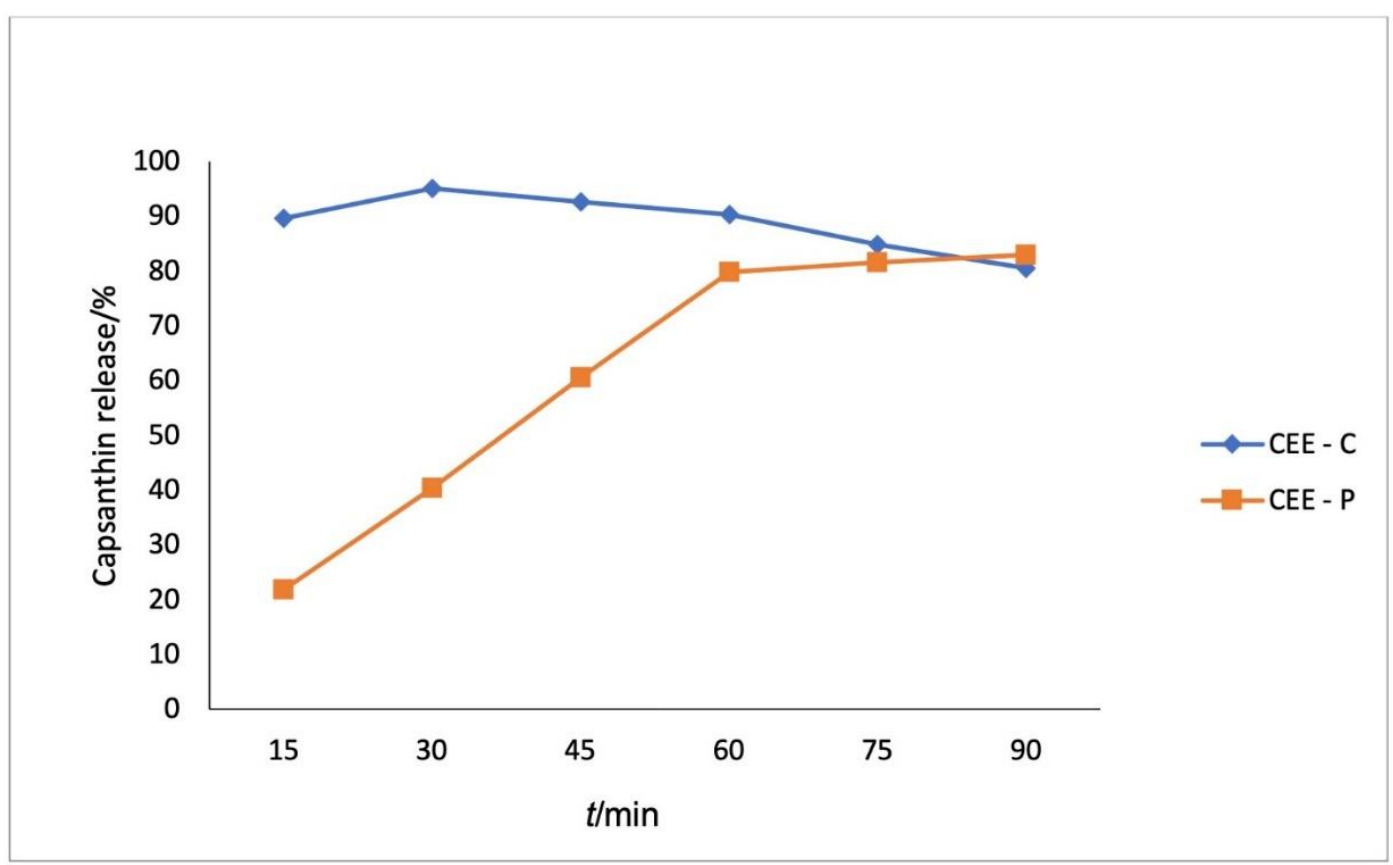

Fig. S2. In vitro drug release of capsanthin enriched extract crystals and capsanthin enriched extract pellets. $\mathrm{CEE}-\mathrm{C}=$ capsanthin enriched extract crystals. $\mathrm{CEE}-\mathrm{P}=$ capsanthin enriched extract pellets 
Please note that this is an unedited version of the manuscript that has been accepted for publication. This version will undergo copyediting and typesetting before its final form for publication. We are providing this version as a service to our readers. The published version will differ from this one as a result of linguistic and technical corrections and layout editing.

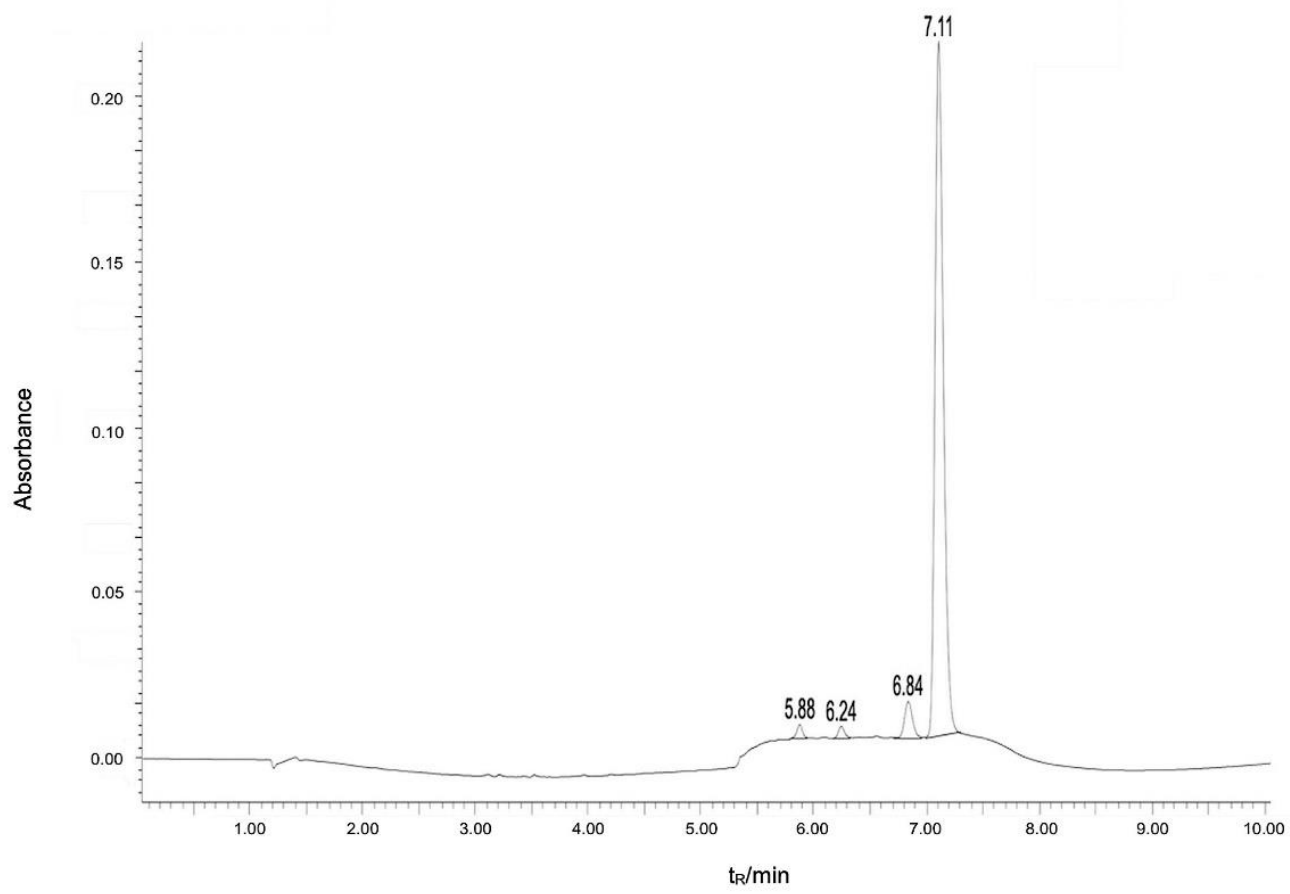

Fig. S3. HPLC chromatogram of capsanthin-enriched extract crystals

\section{Retention time 7.11 corresponds to capsanthin}

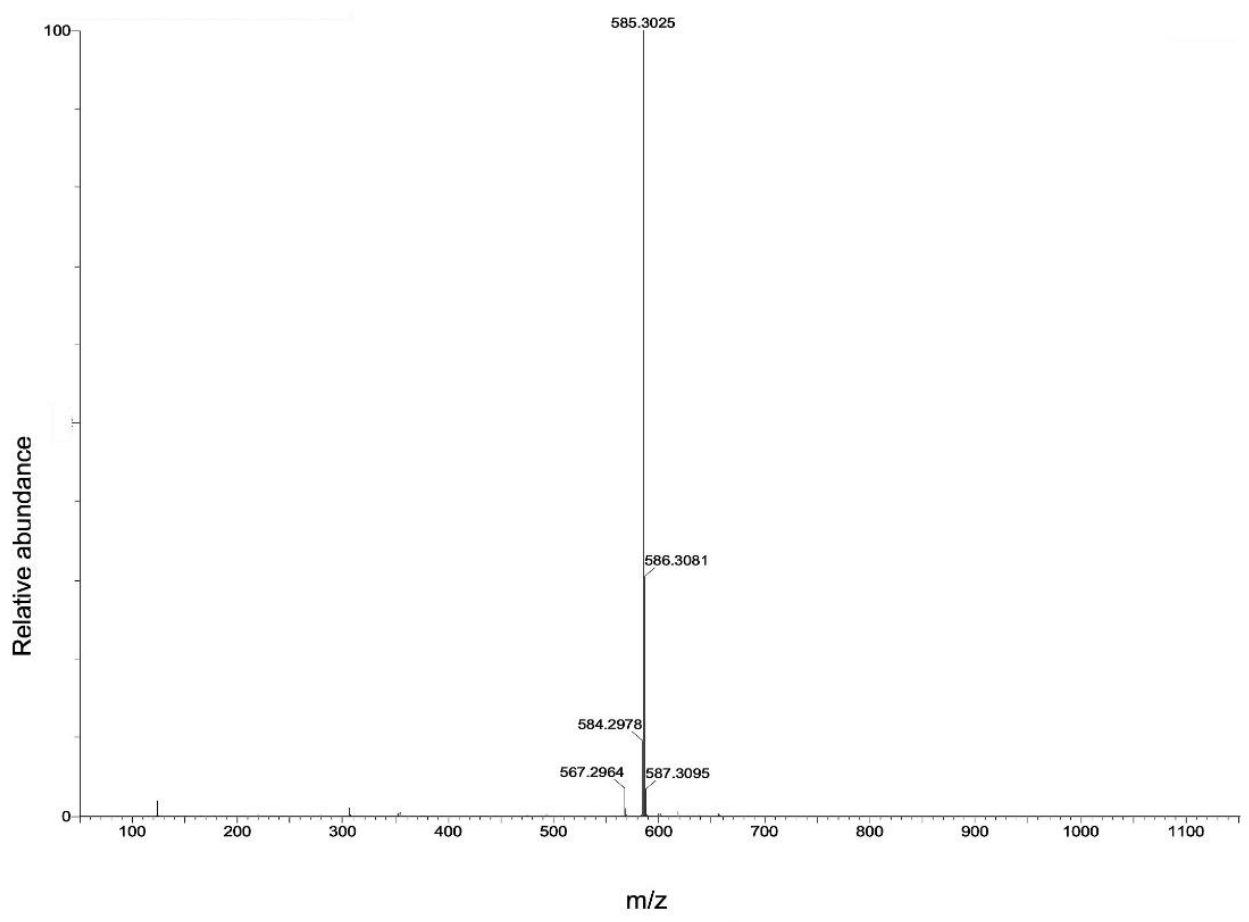

Fig. S4. Mass spectrum of capsanthin. Mass spectrum fragmentation of Retention time 7.122 corresponds to capsanthin 
Please note that this is an unedited version of the manuscript that has been accepted for publication. This version will undergo copyediting and typesetting before its final form for publication. We are providing this version as a service to our readers. The published version will differ from this one as a result of linguistic and technical corrections and layout editing.

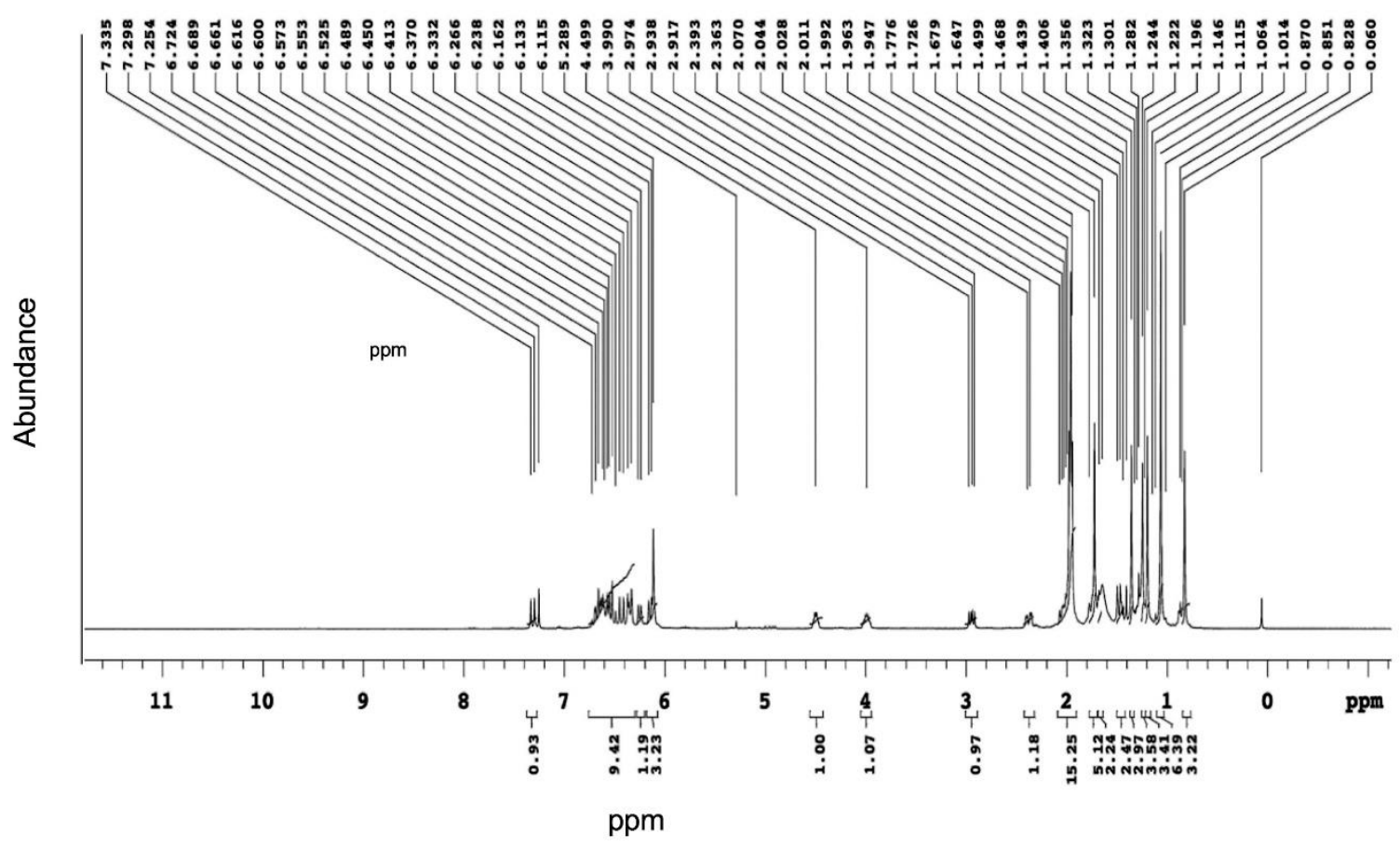

Fig S5 a. ${ }^{1} \mathrm{H}$ NMR spectra of capsanthin

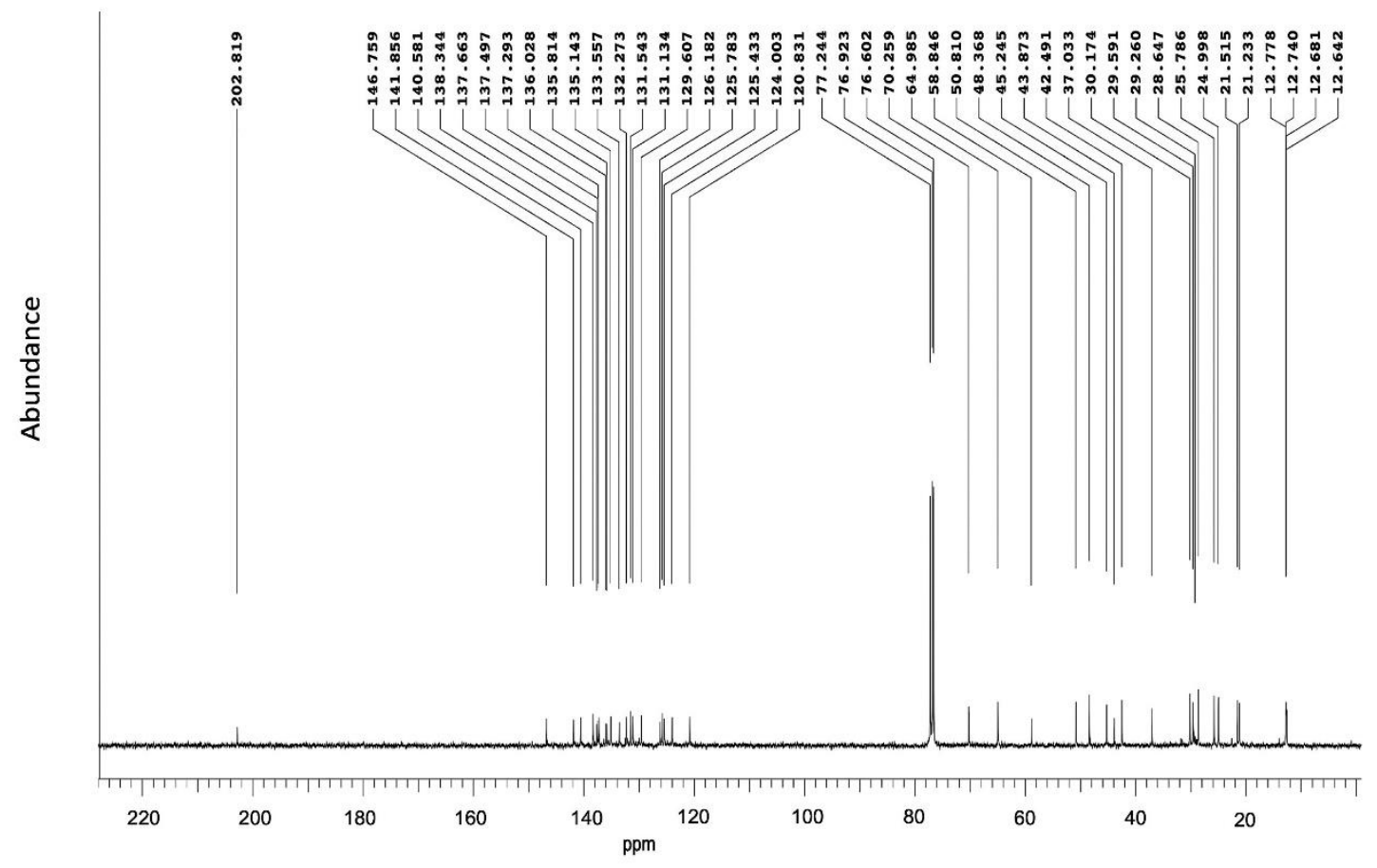

Fig S5 b. ${ }^{13} \mathrm{C}$ NMR spectra of capsanthin 
Please note that this is an unedited version of the manuscript that has been accepted for publication. This version will undergo copyediting and typesetting before its final form for publication. We are providing this version as a service to our readers. The published version will differ from this one as a result of linguistic and technical corrections and layout editing.

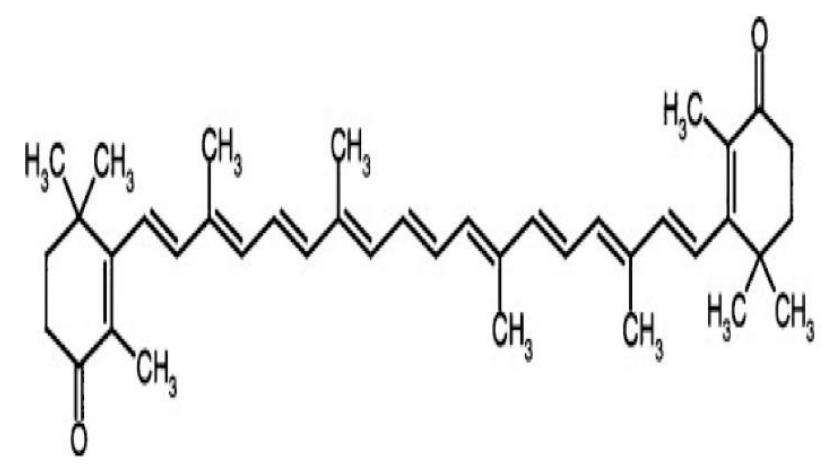

\section{Fig S5 c. Structure of capsanthin}

